Canad. J. Math. Vol. 71 (4), 2019 pp. 749-771

http://dx.doi.org/10.4153/CJM-2018-017-0

(C) Canadian Mathematical Society 2018

\title{
Linear Maps Preserving Matrices of Local Spectral Radius Zero at a Fixed Vector
}

\author{
Abdellatif Bourhim and Constantin Costara
}

Abstract. In this paper, we characterize linear maps on matrix spaces that preserve matrices of local spectral radius zero at some fixed nonzero vector.

\section{Introduction}

Linear preserver problems, in the most general setting, demand the characterization of linear maps between algebras that leave a certain property, a particular relation, or even a subset invariant. This subject is very old and goes back well over a century to the so-called first linear preserver problem, due to Frobenius [30], that determines linear maps preserving the determinant of matrices. The aforesaid Frobenius' work was generalized by J. Dieudonné [28], who characterized linear maps preserving singular matrices. Since then many techniques have been developed to treat preserver problems, and several interesting results have been obtained, notably by Aupetit, Brešar, Jafarian, Molnár, Šemrl, Sourour and others; see, for instance, [2-10,17,21,31,33,35,37-40]. But this topic is still a very active area of research and several problems remain unsolved. One of the most intractable unsolved problems in this area is the famous Kaplansky conjecture, which asserts that every surjective unital invertibility preserving linear map between two semisimple Banach algebras is a Jordan homomorphism. It has not yet fully solved and remains open even for general $C^{*}$-algebras, but it has been confirmed for von Neumann algebras [5] and for the algebra of all bounded linear operators on a Banach spaces $[8,21,33,40]$.

More recently, there has been an upsurge of interest in linear and nonlinear local spectra preserver problems. Bourhim and Ransford were the first ones to consider this type of preserver problem, characterizing in [19] additive maps on the algebra of all linear bounded operators on a complex Banach space $X$ that preserve the local spectrum of operators at each vector of $X$. Their results cleared the way for several authors to describe maps on matrices or operators that preserve local spectrum, local spectral radius, and local inner spectral radius; see, for instance, the last section of the survey article [13] and the references therein. This paper belongs to this subject and investigates the form of linear maps on the algebras of square matrices that preserve matrices of local spectral radius zero at some fixed nonzero vector. Its results generalize, in particular, the main result of [18], where Bourhim and Miller described all linear maps preserving the local spectral radius at a nonzero vector.

Received by the editors September 6, 2017; revised April 13, 2018.

Published electronically August 17, 2018.

AMS subject classification: 47B49, 47A10, 47A11.

Keywords: linear preserver, local spectrum, local spectral radius, matrix. 


\section{Main Results}

Let $\mathcal{L}(X)$ be the algebra of all bounded linear operators on a complex Banach space $X$. The local resolvent set, $\rho_{T}(x)$, of an operator $T \in \mathcal{L}(X)$ at a point $x \in X$ is the union of all open subsets $U$ of $\mathbb{C}$ for which there is an analytic function $\phi: U \rightarrow X$ such that $(T-\lambda) \phi(\lambda)=x,(\lambda \in U)$. The local spectrum of $T$ at $x$ is defined by $\sigma_{T}(x):=\mathbb{C} \backslash \rho_{T}(x)$, and is obviously a closed subset of $\sigma(T)$, the spectrum of $T$. Recall also that the local spectral radius of $T$ at $x \in X$ is defined by

$$
\mathrm{r}_{T}(x):=\limsup _{n \rightarrow+\infty}\left\|T^{n} x\right\|^{\frac{1}{n}} .
$$

In [24], Costara described surjective linear maps on $\mathcal{L}(X)$ that preserve operators of local spectral radius zero at points of $X$. He showed, in particular, that if $\varphi$ is a surjective linear map on $\mathcal{L}(X)$ such that for every $x \in X$ and $T \in \mathcal{L}(X)$, we have

$$
\mathbf{r}_{T}(x)=0 \quad \text { if and only if } \quad \mathbf{r}_{\varphi(T)}(x)=0,
$$

then there exists a nonzero scalar $c$ such that $\varphi(T)=c T$ for all $T \in \mathcal{L}(X)$; see [16] for related results. In this paper, we consider the more general problem of describing linear maps $\varphi$ on $\mathcal{L}(X)$ preserving operators of local spectral radius zero at a nonzero fixed vector $x_{0} \in X$ but when $X=\mathbb{C}^{n}$ is a finite dimensional space. Our aim is to characterize linear maps $\varphi$ on the algebra $\mathcal{M}_{n}$ of all $n \times n$-complex matrices such that

$$
\mathrm{r}_{T}\left(x_{0}\right)=0 \quad \text { if and only if } \quad \mathrm{r}_{\varphi(T)}\left(x_{0}\right)=0 \quad\left(T \in \mathcal{M}_{n}\right) .
$$

Since this problem is trivial for the case when $n=1$, we suppose throughout this paper that $n \geq 2$. For the special case when $n=2$, we obtain the following result.

Theorem 2.1 For a nonzero fixed vector $x_{0} \in \mathbb{C}^{2}$, a linear map $\varphi$ on $\mathcal{M}_{2}$ satisfies (2.1) if and only if there exists a nonzero scalar $\alpha$, an invertible matrix $U \in \mathcal{M}_{2}$ for which $U x_{0}=x_{0}$, and a matrix $Q \in \mathcal{M}_{2}$ satisfying $Q x_{0}=0$ and $\operatorname{tr}(Q) \neq-1$ such that

$$
\varphi(T)=\alpha\left(U T U^{-1}+\operatorname{tr}(T) \cdot Q\right)
$$

for all $T \in \mathcal{M}_{2}$.

In [18], Bourhim and Miller showed that a linear map $\varphi$ on $\mathcal{M}_{n}$ preserves the local spectral radius at a nonzero vector $x_{0} \in \mathbb{C}^{n}$ if and only if $\varphi$ is an automorphism (up to a multiple factor of modulus one) and $x_{0}$ is an eigenvector of the intertwining matrix; see also [23] for nonlinear local spectral radius preservers. For the special case when $n=2$, the above theorem shows that there are nontrivial linear maps on $\mathcal{M}_{2}$ that do not preserve the local spectral radius at $x_{0}$, even after a re-scaling that preserves matrices of local spectral radius zero at $x_{0}$. However, the next result shows that if $n$ is an integer greater than 2 and $\varphi$ is a linear map on $\mathcal{M}_{n}$ satisfying (2.1), then $\varphi$ is, up to a nonzero multiple factor, a local spectral radius preserver at $x_{0}$.

Theorem 2.2 Let $n \geq 3$ be a natural number and fix a nonzero vector $x_{0} \in \mathbb{C}^{n}$. A linear map $\varphi: \mathcal{M}_{n} \rightarrow \mathcal{M}_{n}$ satisfies (2.1) if and only if there exists a nonzero scalar $\alpha$ and an invertible matrix $U \in \mathcal{M}_{n}$ such that $U x_{0}=x_{0}$ and $\varphi(T)=\alpha U T U^{-1}$ for all $T \in \mathcal{M}_{n}$. 
A few comments must be added to the statements of these theorems and the problem considered in this paper. First, let us observe that linear maps $\varphi$ satisfying (2.1) are automatically injective, and therefore bijective. This comes from the fact that a matrix $T_{0} \in \mathcal{M}_{n}$ is trivial if and only if $\mathbf{r}_{T_{0}+T}\left(x_{0}\right)=0$ for all nilpotent matrices $T \in \mathcal{M}_{n}$; see [16, Theorem 3.1]. Indeed, if $\varphi$ is such a map and $\varphi\left(T_{0}\right)=0$ for some matrix $T_{0} \in \mathcal{M}_{n}$, then for every nilpotent matrix $T \in \mathcal{M}_{n}$ we have

$$
0=\mathrm{r}_{\varphi(T)}\left(x_{0}\right)=\mathrm{r}_{\varphi\left(T_{0}\right)+\varphi(T)}\left(x_{0}\right),
$$

and thus $r_{T_{0}+T}\left(x_{0}\right)=0$. Since $T$ is an arbitrary nilpotent matrix, we conclude that $T_{0}=0$ and $\varphi$ is bijective, as claimed. Note that bijectivity of such linear maps $\varphi$ is also confirmed by the conclusion of the previous theorems.

Second, to prove the above theorems, we can and will assume, without loss of generality, that $x_{0}=e_{1}:=(1,0, \ldots, 0)^{t} \in \mathbb{C}^{n}$. Indeed, let $A \in \mathcal{M}_{n}$ be an invertible matrix such that $A x_{0}=e_{1}$. Let $\psi(T):=A \varphi\left(A^{-1} T A\right) A^{-1}$ for $T \in \mathcal{M}_{n}$. Then one can easily check that $\varphi: \mathcal{M}_{n} \rightarrow \mathcal{M}_{n}$ is a linear map such that (2.1) holds if and only if

$$
\mathrm{r}_{T}\left(e_{1}\right)=0 \Longleftrightarrow \mathrm{r}_{\psi(T)}\left(e_{1}\right)=0 \quad\left(T \in \mathcal{M}_{n}\right) .
$$

(This comes from the fact that $\mathrm{r}_{A T A^{-1}}\left(e_{1}\right)=\mathrm{r}_{T}\left(x_{0}\right)$ and $\mathrm{r}_{A^{-1} T A}\left(x_{0}\right)=\mathrm{r}_{T}\left(e_{1}\right)$ for each $\left.T \in \mathcal{M}_{n}\right)$. So, for the remainder of this paper, we shall suppose that

$$
x_{0}=e_{1}=(1,0, \ldots, 0)^{t} \in \mathbb{C}^{n} .
$$

The rest of the paper is organized as follows. Section 2 provides several auxiliary lemmas that will be used in the proof of the main results. Among them, some lemmas give the connection between matrices of local spectrum zero at $x_{0}$ and nilpotent ones. Others provide some permanence properties of maps preserving matrices of local spectral radius zero at $x_{0}$. In particular, we show that any linear map on $\mathcal{M}_{n}$ satisfying (2.1) preserves matrices vanishing at $x_{0}$. In Section 3, we show that, when studying maps $\varphi$ preserving matrices of local spectral radius zero at $x_{0}$, we can additionally assume that $\varphi$ fixes the first column of each matrix in $\mathcal{M}_{n}$. Sections 4 and 5 are designed for the proofs of Theorems 2.1 and 2.2, and finally, we conclude in the last section with some open problems for further research in this area of local spectra preservers.

\section{Preliminary Results}

In this section, we recall some notation and collect some preliminary results that will be used in the proof of the main results. We believe that these auxiliary results are interesting in their own right, and would like to point out that all them remain true for any arbitrary nonzero vector $x_{0} \in \mathbb{C}^{n}$ other than $e_{1}=(1,0, \ldots, 0)^{t}$.

Throughout this paper, let $\mathcal{N}_{n, m}$ be the space of all $n \times m$-complex matrices, and note that $\mathcal{M}_{n}=\mathcal{M}_{n, n}$. For any matrix $T \in \mathcal{M}_{n \times m}$, let $\bar{T}$ be the matrix obtained by taking the complex conjugate of each entry of $T$, and denote, as usual, by $T^{t}$ the transpose of $T$. For a square matrix $T \in \mathcal{M}_{n}$, the spectrum $\sigma(T)$ is the collection of all eigenvalues of $T$, and let $\operatorname{r}(T), \operatorname{det}(T)$ and $\operatorname{tr}(T)$ denote the spectral radius, the determinant and the trace of $T$, respectively. The identity matrix of $\mathcal{M}_{n}$ will be denoted by $I_{n}$. 
We begin with a simple but useful lemma, which will be needed in the proof of Theorem 2.2.

Lemma 3.1 Let $\mu$ be a linear map from $\mathcal{M}_{n}$ into $\mathcal{M}_{1, n}$ such that $\mu(d) c=0$ for all $d \in \mathcal{M}_{n}$ and $c \in \mathcal{M}_{n, 1}$ for which $d c=0$. Then there are $n$ scalars $\beta_{1}, \ldots, \beta_{n} \in \mathbb{C}$ such that

$$
\mu(d)=\beta_{1} d_{1}+\cdots+\beta_{n} d_{n}
$$

for all $d \in \mathcal{M}_{n}$ with rows $d_{1}, \ldots, d_{n} \in \mathcal{M}_{1, n}$.

Proof Let $\mu_{1}, \ldots, \mu_{n}$ be the linear functional components of $\mu$ so that

$$
\mu(d)=\left(\mu_{1}(d), \ldots, \mu_{n}(d)\right)
$$

for all $d \in \mathcal{M}_{n}$. For each $k$, consider the linear function $f_{k}$ on $\mathcal{M}_{n}$ defined by $f_{k}(d):=$ $x_{k 1}$ for all $d=\left(x_{i j}\right)_{i, j} \in \mathcal{M}_{n}$. Let $d=\left(x_{i j}\right)_{i, j} \in \bigcap_{k=1}^{n} \operatorname{ker}\left(f_{k}\right)$ and note that since $d e_{1}=\left(x_{11}, \ldots, x_{n 1}\right)^{t}=0$, we have $\mu(d) e_{1}=\mu_{1}(d)=0$. This shows that $\bigcap_{k=1}^{n} \operatorname{ker}\left(f_{k}\right) \subseteq$ $\operatorname{ker}\left(\mu_{1}\right)$, and thus there are $n$ scalars $\alpha_{11}, \ldots, \alpha_{n 1} \in \mathbb{C}$ such that

$$
\mu_{1}(d)=\sum_{k=1}^{n} \alpha_{1 k} f_{k}(d)=\sum_{k=1}^{n} \alpha_{1 k} x_{k 1}
$$

for all $d=\left(x_{i j}\right)_{i, j} \in \mathcal{M}_{n}$. Analogously, for each $l$, there are $n$ scalars $\alpha_{l 1}, \ldots, \alpha_{l n} \in \mathbb{C}$ such that

$$
\mu_{l}(d)=\sum_{k=1}^{n} \alpha_{l k} x_{k l}
$$

for all $d=\left(x_{i j}\right)_{i, j} \in \mathcal{M}_{n}$.

Now, let $l$ and $s$ be two different integers between 1 and $n$, and set $c:=e_{l}-e_{s}$, where $\left(e_{j}\right)_{1 \leq j \leq n}$ is the canonical basis of $\mathbb{C}^{n}$. Let $d=\left(x_{i j}\right)_{i, j} \in \mathcal{M}_{n}$ be a matrix such that $d c=\left(x_{1 l}-x_{1 s}, \ldots, x_{n l}-x_{n s}\right)^{t}=0$, and note that $\mu(d) c=\mu_{l}(d)-\mu_{s}(d)=0$. Just as above, this implies the existence of $n$ scalars $\gamma_{1}, \ldots, \gamma_{n} \in \mathbb{C}$ such that

$$
\mu_{l}(d)-\mu_{s}(d)=\sum_{k=1}^{n} \gamma_{k}\left(x_{k l}-x_{k s}\right)
$$

for all $d=\left(x_{i j}\right)_{i, j} \in \mathcal{M}_{n}$. This and (3.1) entail that

$$
\sum_{k=1}^{n} \alpha_{l k} x_{k l}-\sum_{k=1}^{n} \alpha_{s k} x_{k s}=\sum_{k=1}^{n} \gamma_{k}\left(x_{k l}-x_{k s}\right)
$$

for all $\left(x_{i j}\right)_{i, j} \in \mathcal{M}_{n}$. Therefore, $\alpha_{l k}=\alpha_{s k}\left(=\gamma_{k}\right)$ for all $k$. Thus, there exist $n$ scalars $\beta_{1}, \ldots, \beta_{n} \in \mathbb{C}$ such that $\alpha_{1 k}=\cdots=\alpha_{n k}=\beta_{k}$ for each $k$, and (3.1) becomes

$$
\mu_{l}(d)=\sum_{k=1}^{n} \beta_{k} x_{k l}
$$

for all $d=\left(x_{i j}\right)_{i, j} \in \mathcal{M}_{n}$. This shows that

$$
\mu(d)=\beta_{1} d_{1}+\cdots+\beta_{n} d_{n}
$$

for all $d \in \mathcal{M}_{n}$ with rows $d_{1}, \ldots, d_{n} \in \mathcal{M}_{1, n}$. The proof is therefore complete. 
For background on local spectral theory, we refer the interested reader to the remarkable books by Aiena [1], and by Laursen and Neumann [34]. However, the local spectra of matrices is well understood and can be found, for instance, in $[18,41]$. For a matrix $T \in \mathcal{M}_{n}$, denote by $\lambda_{1}, \ldots, \lambda_{k}$ the distinct eigenvalues of $T$ and by $N_{1}, \ldots, N_{k}$ the corresponding root spaces. Therefore, $\mathbb{C}^{n}=N_{1} \oplus \cdots \oplus N_{k}$ (algebraic direct sum) and $T=T_{1} \oplus \cdots \oplus T_{k}$, where each $T_{j}$ is the restriction of $T$ to $N_{j}$. Denoting the canonical projection by $P_{j}: \mathbb{C}^{n} \rightarrow N_{j} \subseteq \mathbb{C}^{n}$, the local spectrum of $T$ at any vector $x \in \mathbb{C}^{n}$ is

$$
\sigma_{T}(x)=\bigcup_{1 \leq j \leq k}\left\{\lambda_{j}: P_{j}(x) \neq 0\right\},
$$

and the local spectral radius of $T$ at $x$ is

$$
\mathrm{r}_{T}(x)=\max \left\{\left|\lambda_{j}\right|: 1 \leq j \leq k, P_{j}(x) \neq 0\right\} .
$$

Note that since $P_{1}+\cdots+P_{k}$ is the identity of $\mathbb{C}^{n}$, at least one of the $P_{j}(x)$ 's is nonzero, and thus $\sigma_{T}(x)$ has at least one element.

The following lemma is a simple observation, which shows that if $M$ is an invariant subspace of a matrix $T \in \mathcal{M}_{n}$, then the local spectra of $T$ at any vector of $M$ coincide with the local spectra of $T$ restricted to $M$.

Lemma 3.2 If $M$ is an invariant subspace of a matrix $T \in \mathcal{M}_{n}$, then

$$
\sigma_{T}(x)=\sigma_{\left.T\right|_{M}}(x)
$$

for all $x \in M$.

Proof This comes directly from (3.2) by observing that if the $P_{j}$ 's are as in (3.2), the fact that $M$ is invariant for $T$ implies that $M$ is invariant for each $P_{j}, j=1, \ldots, k$.

The next lemma gives a characterization of matrices of local spectral radius zero at $x_{0}$ in terms of their powers applied to $x_{0}$.

Lemma 3.3 For any matrix $T \in \mathcal{M}_{n}$, we have

$$
\mathrm{r}_{T}\left(x_{0}\right)=0 \Longleftrightarrow T^{n}\left(x_{0}\right)=0 \text {. }
$$

Proof If $T^{n}\left(x_{0}\right)=0$ for some matrix $T \in \mathcal{M}_{n}$, then $T^{k}\left(x_{0}\right)=0$ for all $k \geq n$ and $\mathrm{r}_{T}\left(x_{0}\right)=0$.

Suppose now that $\mathrm{r}_{T}\left(x_{0}\right)=0$ for some matrix $T \in \mathcal{M}_{n}$, and note that (3.3) implies that 0 is an eigenvalue of $T$ and that $x_{0}=P_{1}\left(x_{0}\right) \in N_{1}$, where $N_{1}$ is the root space corresponding to the eigenvalue 0 of $T$ and $P_{1}$ is the canonical projection on $N_{1}$. Since $N_{1}=\operatorname{ker}\left(T^{p}\right)$ for some $p \in \overline{1, n}$, we have $T^{p}\left(x_{0}\right)=0$ and thus $T^{n}\left(x_{0}\right)=0$, as desired.

As a consequence of Lemma 3.3, for a linear map $\varphi: \mathcal{M}_{n} \rightarrow \mathcal{M}_{n}$, we see that (2.1) is equivalent to

$$
T^{n}\left(x_{0}\right)=0 \Longleftrightarrow \varphi(T)^{n}\left(x_{0}\right)=0 \quad\left(T \in \mathcal{M}_{n}\right) .
$$

Of course, if $\mathrm{r}(T)=0$, then $\mathrm{r}_{T}(x)=0$ for each vector $x \in \mathbb{C}^{n}$. A more subtle connection between nilpotent matrices and matrices of local spectral radius zero is 
given by the fact that if $T \in \mathcal{M}_{n}$ and $\left\{x_{0}, T\left(x_{0}\right), \ldots, T^{n-1}\left(x_{0}\right)\right\}$ is a basis of $\mathbb{C}^{n}$, then $\sigma_{T}\left(x_{0}\right)=\sigma(T)$, and in particular $\mathrm{r}_{T}\left(x_{0}\right)=\mathrm{r}(T)$ (see, e.g., [18]). Thus, if the powers of $T$ applied to $x_{0}$ span the whole $\mathbb{C}^{n}$, then $\mathbf{r}_{T}\left(x_{0}\right)=0$ if and only if $T$ is nilpotent.

For any matrix $T \in \mathcal{M}_{n}$, let

$$
n_{T}=\max \left\{k \geq 1: x_{0}, T x_{0}, \ldots, T^{k-1} x_{0} \text { are linearly independent }\right\} \in \overline{1, n} .
$$

Then $\left\{x_{0}, T x_{0}, \ldots, T^{n_{T}-1} x_{0}\right\} \subseteq \mathbb{C}^{n}$ is linearly independent, but $\left\{x_{0}, T x_{0}, \ldots, T^{n_{T}} x_{0}\right\}$ is not, and thus $T^{n_{T}} x_{0}$ is a linear combination of $\left\{x_{0}, T x_{0}, \ldots, T^{n_{T}-1} x_{0}\right\}$. Setting

$$
M_{T}:=\operatorname{Span}\left\{x_{0}, T x_{0}, \ldots, T^{n_{T}-1} x_{0}\right\} \subseteq \mathbb{C}^{n},
$$

we have that $M_{T} \subseteq \mathbb{C}^{n}$ is a nonzero subspace (the cyclic subspace generated by $x_{0}$ ) of dimension $n_{T}$ such that $T\left(M_{T}\right) \subseteq M_{T}$.

The next result gives another connection between matrices of local spectral radius zero at $x_{0}$ and nilpotent ones.

Lemma 3.4 For any matrix $T \in \mathcal{M}_{n}$, we have $\mathrm{r}_{T}\left(x_{0}\right)=0$ if and only if $\mathrm{r}(\widetilde{T})=0$ in $\mathcal{L}\left(M_{T}\right)$, where $M_{T}$ is given by (3.8) and $\widetilde{T}=\left.T\right|_{M_{T}} \in \mathcal{L}\left(M_{T}\right)$.

Proof Since $x_{0} \in M_{T}$ and $T\left(M_{T}\right) \subseteq M_{T}$, from (3.4) we obtain that $r_{T}\left(x_{0}\right)=0$ if and only if $\mathrm{r}_{\widetilde{T}}\left(x_{0}\right)=0$ in $\mathcal{L}\left(M_{T}\right)$. Since $\left\{x_{0}, \widetilde{T} x_{0}, \ldots, \widetilde{T}^{n_{T}-1} x_{0}\right\}$ is a basis of $M_{T}$, in $\mathcal{L}\left(M_{T}\right)$ we have that $\mathrm{r}_{\widetilde{T}}\left(x_{0}\right)=\mathrm{r}(\widetilde{T})$. Thus, $\mathrm{r}_{\widetilde{T}}\left(x_{0}\right)=0$ is equivalent to $\mathrm{r}(\widetilde{T})=0$ in $\mathcal{L}\left(M_{T}\right)$.

The proof of Lemma 3.3 shows that the local spectral radius of a matrix $T \in \mathcal{M}_{n}$ at $x_{0}$ is zero if and only if there is an integer $m$ between 1 and $n$ such that $T^{m} x_{0}=0$. The following lemma shows that if $\mathrm{r}_{T}\left(x_{0}\right)=0$, then $n_{T}$ is the smallest integer $m$ such that $T^{m} x_{0}=0$. Its proof uses the previous lemma.

Lemma 3.5 If $T \in \mathcal{M}_{n}$ is a matrix such that $\mathrm{r}_{T}\left(x_{0}\right)=0$, then $n_{T}$ is the smallest integer $m$ such that $T^{m} x_{0}=0$.

Proof Assume that $T \in \mathcal{M}_{n}$ is a matrix such that $\mathbf{r}_{T}\left(x_{0}\right)=0$, and let $m$ be the smallest integer such that $T^{m} x_{0}=0$. Note that since $x_{0}, T x_{0}, \ldots, T^{m} x_{0}$ are not linearly independent, (3.7) gives $n_{T} \leq m$. To obtain the reverse inequality, keep in mind that $T^{m-1} x_{0} \neq 0$, and let us prove that $x_{0}, T x_{0}, \ldots, T^{m-1} x_{0}$ are linearly independent. If not, we can find a nonzero complex polynomial $P$ with a minimal degree that should be at most $m-1$ such that $P(T) x_{0}=0$. Since $T^{m-1} x_{0} \neq 0$, at least one of the roots of $P$ is not zero. Thus, $P(\lambda)=\prod_{j=1}^{l}\left(\lambda-\lambda_{j}\right) \lambda^{s}$ for some nonzero scalars $\lambda_{j}$ not necessarily distinct, and some integer $s \geq 0$. We have $\prod_{j=1}^{l}\left(T-\lambda_{j} I_{n}\right) T^{s} x_{0}=0$ and

$$
x:=\prod_{j=2}^{l}\left(T-\lambda_{j} I_{n}\right) T^{s} x_{0} \neq 0,
$$

by the minimality of the degree of $P$. Note that $x \in M_{T}$ and $T x=\lambda_{1} x$. Thus, $\operatorname{r}\left(\left.T\right|_{M_{T}}\right)>0$ in $\mathcal{L}\left(M_{T}\right)$, contradicting Lemma 3.4. This contradiction shows that $x_{0}, T x_{0}, \ldots, T^{m-1} x_{0}$ are linearly independent and $m \leq n_{T}$, as desired. 
Lemma 3.4 gives a link between matrices of local spectral radius zero at $x_{0}$ and nilpotent matrices, the main difficulty in using it being now the fact that the subspace $M_{T}$ given by (3.8) changes with $T$. As a corollary of the same lemma, we obtain some information on matrices of the form $x_{0} \otimes f$ in terms of matrices of local spectral radius 0 at $x_{0}$. Recall that if $f$ is a linear functional on $\mathbb{C}^{n}$, then $x_{0} \otimes f$ denotes the rank one operator on $\mathbb{C}^{n}$ defined by

$$
\left(x_{0} \otimes f\right)(x):=f(x) x_{0} \quad\left(x \in \mathbb{C}^{n}\right) .
$$

Lemma 3.6 Let $f$ be a linear functional on $\mathbb{C}^{n}$. Then for every $T \in \mathcal{M}_{n}$ with $\mathrm{r}_{T}\left(x_{0}\right)=$ 0 , we have

$$
\mathrm{r}_{T+x_{0} \otimes f}\left(x_{0}\right)=0 \Longleftrightarrow \mathrm{r}\left(\left.\left(T+x_{0} \otimes f\right)\right|_{M_{T}}\right)=0,
$$

where the spectral radius is computed in $\mathcal{L}\left(M_{T}\right)$, with $M_{T}$ given by (3.8).

Proof By Lemma 3.4, it is sufficient to prove that $M_{T}=M_{T+x_{0} \otimes f}$, and this comes from the fact that

$$
\left(T+x_{0} \otimes f\right)^{j}\left(x_{0}\right) \in T^{j} x_{0}+\operatorname{Span}\left\{x_{0}, T x_{0}, \ldots, T^{j-1} x_{0}\right\}
$$

for each $j \geq 1$.

As a corollary, we obtain the following property for matrices of the form $x_{0} \otimes f$, where $f$ is a linear functional on $\mathbb{C}^{n}$ not vanishing at $x_{0}$.

Lemma 3.7 Let $f$ be a linear functional on $\mathbb{C}^{n}$ such that $f\left(x_{0}\right) \neq 0$. If $T \in \mathcal{M}_{n}$ is a matrix with $\mathbf{r}_{T}\left(x_{0}\right)=0$, then $\mathbf{r}_{T+\lambda x_{0} \otimes f}\left(x_{0}\right) \neq 0$ for all nonzero scalars $\lambda \in \mathbb{C}$.

Proof We shall follow the main idea from [39, Proof of Prop. 2.1]. Let $T \in \mathcal{M}_{n}$ with $\mathbf{r}_{T}\left(x_{0}\right)=0$, and suppose that $\mathbf{r}_{T+\lambda_{0} x_{0} \otimes f}\left(x_{0}\right)=0$ for some $\lambda_{0} \in \mathbb{C} \backslash\{0\}$. Let $M_{T}$ be given by (3.8), and denote $\widetilde{T}:=\left.T\right|_{M_{T}} \in \mathcal{L}\left(M_{T}\right)$. By Lemma 3.6, in $\mathcal{L}\left(M_{T}\right)$ we have $\mathrm{r}(\widetilde{T})=\mathrm{r}\left(\widetilde{T}+\left.\lambda_{0}\left(x_{0} \otimes f\right)\right|_{M_{T}}\right)=0$. For $\lambda \neq 0$, we have $\lambda \notin \sigma(\widetilde{T})$ and $\lambda \notin \sigma\left(\widetilde{T}+\left.\lambda_{0}\left(x_{0} \otimes f\right)\right|_{M_{T}}\right)$, and therefore $f\left((\lambda-\widetilde{T})^{-1} x_{0}\right) \neq 1 / \lambda_{0}$ by [33, Lemma 4$]$. Since $\widetilde{T}^{n_{T}}=0$ in $\mathcal{L}\left(M_{T}\right)$, we then have

$$
f\left(x_{0}\right) / \lambda+\cdots+f\left(T^{n_{T}-1} x_{0}\right) / \lambda^{n_{T}} \neq 1 / \lambda_{0} .
$$

Thus, $-1 / \lambda_{0}+f\left(x_{0}\right) \lambda+\cdots+f\left(T^{n_{T}-1} x_{0}\right) \lambda^{n_{T}} \neq 0$ for every $\lambda \in \mathbb{C}$, and therefore $f\left(x_{0}\right)=\cdots=f\left(T^{n_{T}-1} x_{0}\right)=0$. This contradicts the fact that $f\left(x_{0}\right) \neq 0$, and shows that $\mathbf{r}_{T+\lambda x_{0} \otimes f}\left(x_{0}\right) \neq 0$ for all nonzero scalars $\lambda \in \mathbb{C}$.

We will also need the following simple but useful observation about pencils of matrices having local spectral radius zero at $x_{0}$.

Lemma 3.8 Suppose that $T_{1}, T_{2} \in \mathcal{M}_{n}$ satisfy $\mathrm{r}_{T_{1}+\lambda T_{2}}\left(x_{0}\right)=0$ for all $\lambda \in \mathbb{C}$. Then

$$
T_{1}^{n-1} T_{2} x_{0}+T_{1}^{n-2} T_{2} T_{1} x_{0}+\cdots+T_{1} T_{2} T_{1}^{n-2} x_{0}+T_{2} T_{1}^{n-1} x_{0}=0 .
$$

If, further, $T_{1} x_{0}=0$, then $T_{1}^{n-1} T_{2} x_{0}=0$. 
Proof By (3.5), we have that $\left(T_{1}+\lambda T_{2}\right)^{n} x_{0}=0$ for all $\lambda$. Therefore, the coefficient of $\lambda$ in the development of the left-hand side expression must be zero, and this gives the first equality from the statement. The second one follows immediately from the first.

The following lemma shows that no anti-automorphism on $\mathcal{M}_{n}$ preserves matrices of local spectral radius zero at $x_{0}$.

Lemma 3.9 There exists a matrix $T \in \mathcal{M}_{n}$ such that $T^{t}\left(x_{0}\right)=0$ and $\mathrm{r}_{T}\left(x_{0}\right)>0$.

Proof Let $\left(e_{j}\right)_{1 \leq j \leq n}$ be the canonical basis of $\mathbb{C}^{n}$, and $T \in \mathcal{M}_{n}$ be the matrix given by $T\left(e_{1}\right)=e_{2}, T\left(e_{2}\right)=e_{3}, \ldots, T\left(e_{n-1}\right)=e_{n}$ and $T\left(e_{n}\right)=e_{n}$. By construction, we have $T^{k} x_{0}=e_{n}$ for all $k \geq n$, and thus $\mathrm{r}_{T}\left(x_{0}\right)=\lim \sup _{k \rightarrow \infty}\left\|T^{k} x_{0}\right\|^{\frac{1}{k}}=1$. Moreover, we also have $\left\langle T(x), x_{0}\right\rangle=0$ for all $x \in \mathbb{C}^{n}$. Thus,

$$
\left\langle x, \overline{T^{t}\left(x_{0}\right)}\right\rangle=0 \quad\left(x \in \mathbb{C}^{n}\right),
$$

which gives $T^{t}\left(x_{0}\right)=0$.

The next result shows that linear maps satisfying (2.1) preserve matrices that send $x_{0}$ to $0 \in \mathbb{C}^{n}$. Its proof uses the characterization of maximal subspaces of singular matrices due to Flanders that states that if $\mathcal{S} \subseteq \mathcal{M}_{n}$ is a subspace such that $\operatorname{det} T=0$ for all $T \in \mathcal{S}$, then $\operatorname{dim} \mathcal{S} \leq n(n-1)$. If, however, $\operatorname{dim} \mathcal{S}=n(n-1)$, then there exists a nonzero $x \in \mathbb{C}^{n}$ such that either $\mathcal{S}=\left\{T \in \mathcal{M}_{n}: T(x)=0\right\}$, or $\mathcal{S}=\left\{T \in \mathcal{M}_{n}: T^{t}(x)=0\right\}$; see [29, Theorem 2].

Theorem 3.10 Let $\varphi: \mathcal{M}_{n} \rightarrow \mathcal{M}_{n}$ be a linear map satisfying (2.1). Then for each $T \in \mathcal{M}_{n}$, we have

$$
T\left(x_{0}\right)=0 \Longleftrightarrow \varphi(T)\left(x_{0}\right)=0 .
$$

Proof Since $x_{0}$ is not zero, $\mathcal{S}=\left\{T \in \mathcal{M}_{n}: T\left(x_{0}\right)=0\right\}$ is a subspace of dimension $n(n-1)$. As $\varphi$ is bijective, we note that $\varphi(\mathcal{S}) \subseteq \mathcal{M}_{n}$ is also a subspace of dimension $n(n-1)$. More than that, $\mathrm{r}_{T}\left(x_{0}\right)=0$ for each $T \in \mathcal{S}$; thus, $\mathrm{r}_{\varphi(T)}\left(x_{0}\right)=0$ for each such $T$. Therefore $\varphi(\mathcal{S})$ is a subspace consisting entirely of singular matrices, and thus, by Flanders' result, there is a nonzero vector $x \in \mathbb{C}^{n}$ such that either

$$
\varphi(\mathcal{S})=\left\{T \in \mathcal{M}_{n}: T(x)=0\right\},
$$

or

$$
\varphi(\mathcal{S})=\left\{T \in \mathcal{M}_{n}: T^{t}(x)=0\right\} .
$$

Let us show that (3.11) cannot occur. So, suppose for the sake of contradiction that (3.11) holds, and consider a nonzero vector $y=\left(y_{1}, \ldots, y_{n}\right)^{t} \in \mathbb{C}^{n}$ such that $y \perp x$. Put

$$
T_{0}:=\left[\begin{array}{ccc}
y_{1} \overline{y_{1}} & \cdots & y_{n} \overline{y_{1}} \\
\vdots & \ddots & \vdots \\
y_{1} \overline{y_{n}} & \cdots & y_{n} \overline{y_{n}}
\end{array}\right] \in \mathcal{M}_{n}
$$


and note that $T_{0}^{t}(x)=\langle x, y\rangle y=0$. Therefore, $T_{0} \in \varphi(\mathcal{S})$ and $\mathbf{r}_{T_{0}}\left(x_{0}\right)=0$. As $T_{0}^{n}\left(x_{0}\right)=\left\langle x_{0}, \bar{y}\right\rangle\langle\bar{y}, \bar{y}\rangle^{n-1} \bar{y}$, Lemma 3.3 implies that $\left\langle x_{0}, \bar{y}\right\rangle=0$, and thus $y$ is orthogonal on $\overline{x_{0}}=x_{0}$. That is, $y \perp x$ implies $y \perp x_{0}$, and therefore $x$ and $x_{0}$ must be linearly dependent. This implies that $\varphi(\mathcal{S})=\left\{T \in \mathcal{M}_{n}: T^{t}\left(x_{0}\right)=0\right\}$ and contradicts Lemma 3.9, which ensures the existence of $T \in \varphi(\mathcal{S})$ such that $\mathrm{r}_{T}\left(x_{0}\right) \neq 0$.

Therefore, (3.10) holds and $\varphi(\mathcal{S})=\left\{T \in \mathcal{M}_{n}: T(x)=0\right\}$. If $x$ and $x_{0}$ were linearly independent, then for a matrix $T \in \mathcal{M}_{n}$ satisfying $T\left(x_{0}\right)=x_{0}$ and $T(x)=0$, we would have $T \in \varphi(\mathcal{S})$ and $\mathbf{r}_{T}\left(x_{0}\right)=1$. We arrive at a contradiction, since all the elements in $\varphi(\mathcal{S})$ have local spectral radius at $x_{0}$ equal to zero. Thus, $x=\lambda x_{0}$ for some nonzero scalar $\lambda$, and therefore $\varphi(\mathcal{S})=\mathcal{S}$ and the equivalence in (3.9) is established.

\section{A Reduction}

In this section, we shall prove that, without loss of generality, we can suppose that $\varphi$ fixes the values of matrices at $x_{0}$. That is, we can suppose that $\varphi$ fixes the first column of each matrix in $\mathcal{M}_{n}$.

Let $\varphi: \mathcal{M}_{n} \rightarrow \mathcal{M}_{n}$ be a linear map satisfying (2.1). By using Theorem 3.10, we can reduce its study to the case when

$$
\varphi(T)\left(x_{0}\right)=T\left(x_{0}\right)
$$

for all $T \in \mathcal{M}_{n}$. Indeed, let us first show that there is a nonzero scalar $\alpha \in \mathbb{C}$ such that $\varphi\left(I_{n}\right)\left(x_{0}\right)=\alpha x_{0}$. If not, since $\varphi$ is surjective, one can find $T \in \mathcal{M}_{n}$ such that

$$
\varphi(T)\left(x_{0}\right)=\varphi\left(I_{n}\right)\left(x_{0}\right) \quad \text { and } \quad \varphi(T)\left(\varphi\left(I_{n}\right)\left(x_{0}\right)\right)=0 .
$$

It then follows that $\varphi\left(T-I_{n}\right)\left(x_{0}\right)=0$ and $\mathrm{r}_{\varphi\left(T-I_{n}\right)}\left(x_{0}\right)=0$. But since $\varphi$ satisfies (2.1), we have $\mathrm{r}_{T-I_{n}}\left(x_{0}\right)=0$, and therefore, directly from the definition of the local spectral radius, we get $\mathbf{r}_{T}\left(x_{0}\right)=1$. In particular, $\mathbf{r}_{T}\left(x_{0}\right) \neq 0$, and $(2.1)$ gives $\mathbf{r}_{\varphi(T)}\left(x_{0}\right) \neq 0$. This contradicts the fact that $\varphi(T)^{2}\left(x_{0}\right)=0$ and shows that $\varphi\left(I_{n}\right)\left(x_{0}\right)=\alpha x_{0}$ for some complex number $\alpha$ which, by (3.9), must not be zero, as claimed.

Next, we show that there is an invertible matrix $U \in \mathcal{M}_{n}$ such that $U\left(x_{0}\right)=\alpha x_{0}$ and $\varphi(T)\left(x_{0}\right)=U T\left(x_{0}\right)$ for all $T \in \mathcal{M}_{n}$. Indeed, let $\varphi=\left(\varphi_{i j}\right)_{1 \leq i, j \leq n}$ be the linear functionals defined to be the entries of $\varphi$, and let us show that there are $n$ scalar $\alpha_{11}, \alpha_{12}, \ldots, \alpha_{1 n} \in \mathbb{C}$ such that

$$
\varphi_{11}(T)=\alpha_{11} x_{11}+\alpha_{12} x_{21}+\cdots+\alpha_{1 n} x_{n 1}
$$

for all $T=\left(x_{i j}\right)_{1 \leq i, j \leq n} \in \mathcal{M}_{n}$. To do so, note that there exist $\left(\beta_{i j}\right)_{1 \leq i, j \leq n}$ such that $\varphi_{11}(T)=\sum_{i, j} \beta_{i j} x_{i j}$ for all $T=\left(x_{i j}\right)_{1 \leq i, j \leq n} \in \mathcal{M}_{n}$. In particular, for any $T=\left(x_{i j}\right)_{1 \leq i, j \leq n} \in \mathcal{M}_{n}$ for which $T x_{0}=\left(x_{11}, x_{21}, \ldots\right)^{t}=0$, the identity (3.9)) implies that $\varphi_{11}(T)=\sum_{1 \leq i, j \leq n, j \neq 1} \beta_{i j} x_{i j}=0$. This tells us that $\beta_{i j}=0$ for all $1 \leq i, j \leq n$ such that $j \neq 1$, and thus (4.1) is satisfied with $\alpha_{1 i}=\beta_{i 1}$ for all $i$. Similarly, for every $i$, there are $n$ scalars $\alpha_{i 1}, \alpha_{i 2}, \ldots, \alpha_{i n} \in \mathbb{C}$ such that

$$
\varphi_{i 1}(T)=\alpha_{i 1} x_{11}+\alpha_{i 2} x_{21}+\cdots+\alpha_{i n} x_{n 1}
$$


for all $T=\left(x_{i j}\right)_{1 \leq i, j \leq n} \in \mathcal{M}_{n}$. Now, set $U:=\left(\alpha_{i j}\right)_{i, j}$ and note that

$$
\varphi(T) x_{0}=\left(\begin{array}{c}
\varphi_{11}(T) \\
\varphi_{21}(T) \\
\vdots \\
\varphi_{n 1}(T)
\end{array}\right)=\left(\begin{array}{c}
\alpha_{11} x_{11}+\alpha_{12} x_{21}+\cdots \\
\alpha_{21} x_{11}+\alpha_{22} x_{21}+\cdots \\
\vdots \\
\alpha_{n 1} x_{11}+\alpha_{n 2} x_{21}+\cdots
\end{array}\right)=U T x_{0}
$$

for all $T=\left(x_{i j}\right)_{1 \leq i, j \leq n} \in \mathcal{M}_{n}$. That $\varphi$ is bijective implies that the functionals from the first column of $\varphi$ are linearly independent, and therefore $U$, is invertible. That $\varphi\left(I_{n}\right)\left(x_{0}\right)=\alpha x_{0}$ implies $U\left(x_{0}\right)=\alpha x_{0}$, with $\alpha \neq 0$; as desired.

Now, put

$$
\psi(T):=\frac{1}{\alpha} U^{-1} \varphi(T) U \quad\left(T \in \mathcal{M}_{n}\right) .
$$

We have $\mathrm{r}_{\psi(T)}\left(x_{0}\right)=\frac{1}{|\alpha|} \mathrm{r}_{\varphi(T)}\left(x_{0}\right)$ for every $T \in \mathcal{M}_{n}$, and thus $\psi$ satisfies (2.1). Moreover, we have

$$
\begin{aligned}
\psi(T)\left(x_{0}\right) & =\left(U^{-1} \varphi(T)(U / \alpha)\right)\left(x_{0}\right)=U^{-1} \varphi(T)\left(x_{0}\right) \\
& =T\left(x_{0}\right)
\end{aligned}
$$

for all $T \in \mathcal{M}_{n}$, and $\psi$ does not change the values of matrices at $x_{0}$.

Therefore, for the remainder of the paper, we shall suppose that $\varphi: \mathcal{M}_{n} \rightarrow \mathcal{M}_{n}$ is a linear map satisfying (2.1) such that $\varphi(T)\left(x_{0}\right)=T\left(x_{0}\right)$ for all $T \in \mathcal{M}_{n}$. Write $T \in \mathcal{M}_{n}$ as

$$
T=\left(\begin{array}{ll}
a & b \\
c & d
\end{array}\right)
$$

with $a \in \mathbb{C}, b \in \mathcal{M}_{1, n-1}, c \in \mathcal{M}_{n-1,1}$, and $d \in \mathcal{M}_{n-1}$. Then $\varphi(T)$ is of the form

$$
\varphi(T)=\left(\begin{array}{ll}
a & B(T) \\
c & D(T)
\end{array}\right),
$$

with $B: \mathcal{M}_{n} \rightarrow \mathcal{M}_{1, n-1}$ and $D: \mathcal{M}_{n} \rightarrow \mathcal{M}_{n-1}$ linear maps, which are to be determined.

It is worth pointing out that when proving Theorem 2.1, it suffices to show that a linear map $\varphi$ on $\mathcal{M}_{2}$ of the form (4.2) satisfies (2.1) if and only if there is a matrix $Q \in \mathcal{M}_{2}$ satisfying $Q x_{0}=0$ and $\operatorname{tr}(Q) \neq-1$ such that

$$
\varphi(T)=T+\operatorname{tr}(T) \cdot Q
$$

for all $T \in \mathcal{M}_{2}$. However, when $n \geq 3$, we shall prove that if a linear map on $\mathcal{M}_{n}$ of the form (4.2) satisfies (2.1), then $\varphi$ must be the identity on $\mathcal{M}_{n}$.

\section{Proof of Theorem 2.1}

Assume that there exists a matrix $Q \in \mathcal{M}_{2}$ satisfying $Q x_{0}=0$ and $\operatorname{tr}(Q) \neq-1$ such that $\varphi$ has the form (4.3). Note that since $Q x_{0}=0$ and $\operatorname{tr}(Q) \neq-1$, the matrix $Q$ must be of the form

$$
Q=\left(\begin{array}{ll}
0 & \alpha \\
0 & \beta
\end{array}\right)
$$


for some scalars $\alpha$ and $\beta$ with $\beta \neq-1$. Given $T=\left(\begin{array}{ll}a & b \\ c & d\end{array}\right) \in \mathcal{M}_{2}$, it then follows that

$$
\varphi(T)=\left(\begin{array}{ll}
a & b+\alpha(a+d) \\
c & d+\beta(a+d)
\end{array}\right) .
$$

Note that since $\beta \neq-1$, we see that $\operatorname{tr}(T)=0$ if and only if $\operatorname{tr}(\varphi(T))=0$. Therefore, Lemma 3.3 tells us that

$$
\begin{aligned}
\mathrm{r}_{T}\left(x_{0}\right)=0 & \Longleftrightarrow a^{2}+b c=a c+c d=0 \\
& \Longleftrightarrow a=c=0 \text { or } a^{2}+b c=\operatorname{tr}(T)=0 \\
& \Longleftrightarrow a=c=0 \text { or } a^{2}+b c=\operatorname{tr}(\varphi(T))=0 \\
& \Longleftrightarrow \mathrm{r}_{\varphi(T)}\left(x_{0}\right)=0,
\end{aligned}
$$

and then (2.1) is satisfied.

Conversely, suppose that $\varphi: \mathcal{M}_{2} \rightarrow \mathcal{M}_{2}$ is a linear map of the form (4.2) such that (2.1) holds for $x_{0}=(1,0)^{t} \in \mathbb{C}^{2}$. By (3.6), for $T=\left(\begin{array}{ll}a & b \\ c & d\end{array}\right) \in \mathcal{M}_{2}$, we have

$$
a^{2}+b c=c(a+d)=0 \Longleftrightarrow a^{2}+B(T) c=c(a+D(T))=0 .
$$

Write

$$
\begin{aligned}
& B(T):=\alpha_{1} a+\alpha_{2} b+\alpha_{3} c+\alpha_{4} d, \\
& D(T):=\beta_{1} a+\beta_{2} b+\beta_{3} c+\beta_{4} d
\end{aligned}
$$

for all $T=\left(\begin{array}{ll}a & b \\ c & d\end{array}\right) \in \mathcal{M}_{2}$. Taking $a=b=d=0$ and $c=1$, it then follows from (5.1)-(5.3) that $\alpha_{3}=\beta_{3}=0$. Now given any $a \neq 0$ and $b \neq 0$, for $d=-a$ and $c \neq 0$ such that $a^{2}+b c=0$, from (5.1)-(5.3), we infer that

$$
\left(\alpha_{1}-\alpha_{4}\right) a+\left(\alpha_{2}-1\right) b=\left(1+\beta_{1}-\beta_{4}\right) a+\beta_{2} b=0 .
$$

Since this holds for all $a, b \neq 0$, then $\alpha_{1}=\alpha_{4}, \alpha_{2}=1, \beta_{4}=1+\beta_{1}$ and $\beta_{2}=0$. Thus,

$$
\varphi(T)=\left(\begin{array}{cc}
a & b+\alpha(a+d) \\
c & \beta a+(1+\beta) d
\end{array}\right) \quad\left(T=\left(\begin{array}{ll}
a & b \\
c & d
\end{array}\right) \in \mathcal{M}_{2}\right)
$$

for some $\alpha, \beta \in \mathbb{C}$. Since $\varphi$ is bijective, its image in $\mathcal{M}_{2}$ contains matrices of nonzero trace, and therefore $\beta \neq-1$. Denoting

$$
Q=\left(\begin{array}{ll}
0 & \alpha \\
0 & \beta
\end{array}\right) \in \mathcal{M}_{2}
$$

then

$$
\varphi(T)=T+\operatorname{tr}(T) \cdot Q \quad\left(T \in \mathcal{M}_{2}\right),
$$

where $Q \in \mathcal{M}_{2}$ satisfies $Q x_{0}=0$ and $\operatorname{tr}(Q) \neq-1$.

\section{Proof of Theorem 2.2}

In what follows, suppose that $n>2$ and note that the "if" part of Theorem 2.2 is obvious. For the "only if" part, assume that $\varphi$ is of the form (4.2) and satisfies (2.1), and then let us show that $\varphi$ is the identity of $\mathcal{M}_{n}$. We shall prove this in several steps. 
Step 1 There is $R_{0}=\left(\begin{array}{cc}a_{0} & b_{0} \\ 0 & 0\end{array}\right) \in \mathcal{M}_{n}$ with $a_{0} \in \mathbb{C} \backslash\{0\}$ and $b_{0} \in \mathcal{M}_{1, n-1}$ such that $B\left(R_{0}\right) \neq 0$.

Let us suppose, for a contradiction, that $B(R)=0$ for every $R=\left(\begin{array}{ll}a & b \\ 0 & 0\end{array}\right) \in \mathcal{M}_{n}$ with $a \in \mathbb{C}$ and $b \in \mathcal{M}_{1, n-1}$, and keep in mind that (4.2) entails that

$$
\varphi(R)=\left(\begin{array}{cc}
a & 0 \\
0 & D(R)
\end{array}\right)
$$

for each such $R$. If $D(R) \notin \mathbb{C} I_{n-1}$ for some $a \in \mathbb{C} \backslash\{0\}$ and $b \in \mathcal{M}_{1, n-1}$, then there exists linearly independent vectors $x, y \in \operatorname{Span}\left\{e_{2}, \ldots, e_{n}\right\}$ such that $\varphi(R) x=y$. Then $\left\{x_{0}, x, y\right\}$ is linearly independent in $\mathbb{C}^{n}$ with $\varphi(R) x_{0}=a x_{0}, \varphi(R) x=y$ and $\varphi(R) y \in \operatorname{Span}\left\{e_{2}, \ldots, e_{n}\right\}$. Let $T \in \mathcal{M}_{n}$ such that

$$
\varphi(T) x_{0}=-a x_{0}+x, \varphi(T) x=-a^{2} x_{0}+a x \quad \text { and } \quad \varphi(T) y=a^{3} x_{0}-a y-\varphi(R) y .
$$

Then

$$
\varphi(T)^{2} x_{0}=\varphi(T)\left(-a x_{0}+x\right)=-a\left(-a x_{0}+x\right)+\left(-a^{2} x_{0}+a x\right)=0,
$$

and thus $\mathrm{r}_{\varphi(T)}\left(x_{0}\right)=0$, which implies that $\mathrm{r}_{T}\left(x_{0}\right)=0$. Now, Lemma 3.7 gives $\mathrm{r}_{T+R}\left(x_{0}\right) \neq 0$, and consequently, $\mathrm{r}_{\varphi(T)+\varphi(R)}\left(x_{0}\right) \neq 0$. But since

$$
\begin{aligned}
(\varphi(T)+\varphi(R))^{3} x_{0} & =(\varphi(T)+\varphi(R))^{2} x \\
& =(\varphi(T)+\varphi(R))\left(-a^{2} x_{0}+a x+y\right) \\
& =\varphi(T)\left(-a^{2} x_{0}+a x+y\right)+\varphi(R)\left(-a^{2} x_{0}+a x+y\right) \\
& =\left(a^{3} x_{0}-a y-\varphi(R) y\right)+\left(-a^{3} x_{0}+a y+\varphi(R) y\right) \\
& =0,
\end{aligned}
$$

we arrive at a contradiction. Therefore, $D(R) \in \mathbb{C} I_{n-1}$ for every $a \in \mathbb{C} \backslash\{0\}$ and $b \in$ $\mathcal{M}_{1, n-1}$, and then by continuity $D(R) \in \mathbb{C} I_{n-1}$ for all $a \in \mathbb{C}$ and $b \in \mathcal{M}_{1, n-1}$. This means that the image under $\varphi$ of the subspace of all matrices of the form $\left.\left(\begin{array}{ll}a & b \\ 0 & 0\end{array}\right)\right) \in \mathcal{M}_{n}$ with $a \in \mathbb{C}, b \in \mathcal{M}_{1, n-1}$, is of dimension at most 2. This contradicts the fact that $\varphi$ is bijective, and completes the proof of this step.

Step 2 For every $R=\left(\begin{array}{ll}a & b \\ 0 & 0\end{array}\right) \in \mathcal{M}_{n}$ with $a \in \mathbb{C}$ and $b \in \mathcal{M}_{1, n-1}$, we have $D(R) \in \mathbb{C I}_{n-1}$, and thus

$$
\varphi(R)=\left(\begin{array}{cc}
a & B(R) \\
0 & \gamma(R) I_{n-1}
\end{array}\right)
$$

with $\gamma(R) \in \mathbb{C}$.

By Step 1, there is $R_{0}=\left(\begin{array}{cc}a_{0} & b_{0} \\ 0 & 0\end{array}\right) \in \mathcal{M}_{n}$ with $a_{0} \in \mathbb{C} \backslash\{0\}$ and $b_{0} \in \mathcal{M}_{1, n-1}$ such that $B\left(R_{0}\right) \neq 0$. Since $D$ is linear, it suffices to show that $D(R) \in \mathbb{C} I_{n-1}$ for all $R=\left(\begin{array}{ll}a & b \\ 0 & 0\end{array}\right) \epsilon$ $\mathcal{M}_{n}$ for which $a$ is sufficiently close to $a_{0}$ and $b=\left(\alpha_{2}, \ldots, \alpha_{n}\right)$ is sufficiently close to $b_{0}$ so that $a \neq 0$ and $B(R) \neq 0$. Consider such a matrix $R$, and note that $R$ is of the form $x_{0} \otimes f$, where $f$ is a linear functional on $\mathbb{C}^{n}$. Let $T \in \mathcal{M}_{n}$ be a matrix such that 
$\mathrm{r}_{T}\left(x_{0}\right)=0$, and then Lemma 3.7 implies that $\mathrm{r}_{\varphi^{-1}(T)+\lambda x_{0} \otimes f}\left(x_{0}\right)=\mathrm{r}_{\varphi^{-1}(T)+\lambda R}\left(x_{0}\right) \neq 0$ for all nonzero scalars $\lambda \in \mathbb{C}$. Therefore,

$$
\mathrm{r}_{T+\lambda \varphi(R)}\left(x_{0}\right) \neq 0
$$

for all nonzero scalars $\lambda \in \mathbb{C}$.

Now, suppose that there exists $c \in \mathbb{C}^{n-1}$ such that $\{c, D(R) c\}$ is a linearly independent system. By continuity, one can find $c$ such that $B(R) c \neq 0$. That $\{c, D(R) c\}$ is a linearly independent system implies that $\{c, a c+D(R) c\}$ is also a linearly independent system. Set $\lambda_{0}:=-B(R) c / a^{2} \in \mathbb{C} \backslash\{0\}$ so that

$$
\lambda_{0} a^{2}+B(R) c=0,
$$

and consider then $d \in \mathcal{M}_{n-1}$ such that

$$
d(c)=-\lambda_{0}(a c+D(R) c) \text { and } d(a c+D(R) c)=0 .
$$

Let $T:=\left(\begin{array}{ll}0 & 0 \\ c & d\end{array}\right) \in \mathcal{M}_{n}$, and note that, in view of (6.3), we have $d^{2}(c)=0$. It then follows that $T^{3}=\left(\begin{array}{ll}0 & 0 \\ 0 & d^{3}\end{array}\right)$, and thus $\mathbf{r}_{T}\left(x_{0}\right)=0$. By (6.2) and (6.3), we also have

$$
\left(T+\lambda_{0} \varphi(R)\right)^{2}=\left(\begin{array}{cc}
\lambda_{0} a & \lambda_{0} B(R) \\
c & d+\lambda_{0} D(R)
\end{array}\right)^{2}=\left(\begin{array}{cc}
\lambda_{0}\left(\lambda_{0} a^{2}+B(R) c\right) & * \\
\lambda_{0}(a c+D(R) c)+d c & *
\end{array}\right)=\left(\begin{array}{cc}
0 & * \\
0 & *
\end{array}\right),
$$

and therefore $\left(T+\lambda_{0} \varphi(R)\right)^{2}\left(x_{0}\right)=0$. This contradicts (6.1) and shows that $D(R) \epsilon$ $\mathbb{C} I_{n-1}$, as desired.

Step 3 There is a nonzero scalar $\alpha \in \mathbb{C}$ such that

$$
\varphi\left(\begin{array}{ll}
0 & b \\
0 & 0
\end{array}\right)=\left(\begin{array}{cc}
0 & \alpha b \\
0 & 0
\end{array}\right) \quad\left(b \in \mathcal{M}_{1, n-1}\right)
$$

By Step 2, there are two linear maps $u: \mathcal{M}_{1, n-1} \rightarrow \mathcal{M}_{1, n-1}$ and $\gamma: \mathcal{M}_{1, n-1} \rightarrow \mathbb{C}$ such that

$$
\varphi\left(\begin{array}{ll}
0 & b \\
0 & 0
\end{array}\right)=\left(\begin{array}{cc}
0 & u(b) \\
0 & \gamma(b) I_{n-1}
\end{array}\right) \quad\left(b \in \mathcal{M}_{1, n-1}\right) .
$$

Let us also write

$$
\varphi\left(\begin{array}{ll}
0 & 0 \\
c & 0
\end{array}\right)=\left(\begin{array}{cc}
0 & v(c) \\
c & w(c)
\end{array}\right) \quad\left(c \in \mathcal{M}_{n-1,1}\right)
$$

with $v: \mathcal{M}_{n-1,1} \rightarrow \mathcal{M}_{1, n-1}$ and $w: \mathcal{M}_{n-1,1} \rightarrow \mathcal{M}_{n-1}$ linear maps. Let $T=\left(\begin{array}{ll}0 & b \\ c & 0\end{array}\right) \in \mathcal{M}_{n}$, and let us first show that $b c=0$ if and only if

$$
\mathbb{C}^{n} \ni 0=\left(\varphi\left(\begin{array}{cc}
0 & \lambda b \\
c & 0
\end{array}\right)\right)^{n} x_{0}=\left(\left(\begin{array}{cc}
0 & v(c) \\
c & w(c)
\end{array}\right)+\lambda\left(\begin{array}{cc}
0 & u(b) \\
0 & \gamma(b) I_{n-1}
\end{array}\right)\right)^{n} x_{0}
$$

for all $\lambda \in \mathbb{C}$. Indeed, since

$$
\left(\begin{array}{ll}
0 & b \\
c & 0
\end{array}\right)^{2 k}=\left(\begin{array}{cc}
(b c)^{k} & 0 \\
0 & (c b)^{k}
\end{array}\right) \quad(k \geq 1)
$$


it then follows that $\mathbf{r}_{T}\left(x_{0}\right)=0$ if and only if $b c=0$. Take any scalar $\lambda \in \mathbb{C}$ and replace $b$ by $\lambda b$; it then follows from this and (3.6) that $b c=0$ if and only if (6.6) holds, as claimed.

Next, we show that $\gamma(\cdot)$ is identically zero. Let $b \in \mathcal{M}_{1, n-1}$, and pick up a nonzero $c \in \mathcal{M}_{n-1,1}$ such that $b c=0$. Set

$$
T_{1}:=\left(\begin{array}{cc}
0 & u(b) \\
0 & \gamma(b) I_{n-1}
\end{array}\right) \quad \text { and } \quad T_{2}:=\left(\begin{array}{cc}
0 & v(c) \\
c & w(c)
\end{array}\right)
$$

and note that (6.6) implies that $\mathrm{r}_{T_{1}+\lambda T_{2}}\left(x_{0}\right)=0$ for all $\lambda \in \mathbb{C}$. Since $T_{1} x_{0}=0$, Lemma 3.8 gives

$$
0=T_{1}^{n-1} T_{2} x_{0}=\left(\begin{array}{cc}
0 & u(b) \\
0 & \gamma(b) I_{n-1}
\end{array}\right)^{n-1}\left(\begin{array}{cc}
0 & v(c) \\
c & w(c)
\end{array}\right) x_{0}
$$

that is

$$
0=\left(\begin{array}{cc}
0 & \gamma(b)^{n-2} u(b) \\
0 & \gamma(b)^{n-1} I_{n-1}
\end{array}\right)\left(\begin{array}{l}
0 \\
c
\end{array}\right)=\left(\begin{array}{c}
\gamma(b)^{n-2} u(b) c \\
\gamma(b)^{n-1} c
\end{array}\right) .
$$

This implies $\gamma(b)^{n-1} c=0$, but, since $c \neq 0$, we see that $\gamma(b)=0$. Therefore, $\gamma$ is identically zero, as claimed.

Finally, let us show that there is a nonzero scalar $\alpha \in \mathbb{C}$ such that $u(b)=\alpha b$ for all $b \in \mathcal{M}_{1, n-1}$. By what has been proved before, (6.5) becomes

$$
\varphi\left(\begin{array}{ll}
0 & b \\
0 & 0
\end{array}\right)=\left(\begin{array}{cc}
0 & u(b) \\
0 & 0
\end{array}\right) \quad\left(b \in \mathcal{M}_{1, n-1}\right) .
$$

Let $b \in \mathcal{M}_{1, n-1}$ and $c \in \mathcal{M}_{n-1,1}$ such that $b c=0$, and let us prove that $u(b) c=0$. Let $\lambda \in \mathbb{C}$ and note that (6.6) becomes

$$
\left(\left(\begin{array}{cc}
0 & v(c) \\
c & w(c)
\end{array}\right)+\lambda\left(\begin{array}{cc}
0 & u(b) \\
0 & 0
\end{array}\right)\right)^{n} x_{0}=0
$$

Denote

$$
M=\operatorname{Span}\left\{\left(\begin{array}{ll}
0 & v(c) \\
c & w(c)
\end{array}\right)^{k} x_{0}: k \geq 0\right\} \subseteq \mathbb{C}^{n},
$$

and note that, since the range of $\left(\begin{array}{cc}0 & u(b) \\ 0 & 0\end{array}\right)$ is in $\mathbb{C} x_{0}$, we have

$$
M=\operatorname{Span}\left\{\left(\left(\begin{array}{cc}
0 & v(c) \\
c & w(c)
\end{array}\right)+\lambda\left(\begin{array}{cc}
0 & u(b) \\
0 & 0
\end{array}\right)\right)^{k} x_{0}: k \geq 0\right\} .
$$

Therefore, $\left(\begin{array}{ll}0 & v(c) \\ c & w(c)\end{array}\right)+\lambda\left(\begin{array}{cc}0 & u(b) \\ 0 & 0\end{array}\right)$ sends $M$ into $M$, and is nilpotent in $\mathcal{L}(M)$, in view of (6.7). In particular, this implies that the trace of

$$
\left.\left.\left(\begin{array}{cc}
0 & u(b) \\
0 & 0
\end{array}\right)\right|_{M}\left(\begin{array}{ll}
0 & v(c) \\
c & w(c)
\end{array}\right)\right|_{M}
$$

in $\mathcal{L}(M)$ is zero. Now observe that

$$
\left.\left.\left(\begin{array}{cc}
0 & u(b) \\
0 & 0
\end{array}\right)\right|_{M}\left(\begin{array}{cc}
0 & v(c) \\
c & w(c)
\end{array}\right)\right|_{M}=\left.\left(\begin{array}{cc}
u(b) c & u(b) w(c) \\
0 & 0
\end{array}\right)\right|_{M}
$$

and therefore its trace is $u(b) c=0$, as desired. 
Therefore, given any $b \in \mathcal{M}_{1, n-1}$ there exists $\lambda_{b} \in \mathbb{C}$ such that $u(b)=\lambda_{b} b$. The linearity of $u$ implies the existence of $\alpha \in \mathbb{C}$ such that $u(b)=\alpha b$ for all $b \in \mathcal{M}_{1, n-1}$. That $\varphi$ is injective gives $\alpha \neq 0$. We have therefore proved the existence of a nonzero complex scalar $\alpha$ such that (6.4) holds, and the proof of this step is complete.

Step 4 For each $T \in \mathcal{M}_{n}$, we have

$$
T^{2}\left(x_{0}\right)=0 \Longleftrightarrow \varphi(T)^{2}\left(x_{0}\right)=0 .
$$

Let $T \in \mathcal{M}_{n}$, and note that, since $\varphi$ is bijective and $\varphi^{-1}$ also satisfies (2.1), it suffices to prove that $T^{2} x_{0}=0$ implies $\varphi(T)^{2} x_{0}=0$. So, assume that $T^{2} x_{0}=0$ and suppose, for a contradiction, that $\varphi(T)^{2}\left(x_{0}\right) \neq 0$. Since $\varphi(T) x_{0}=T x_{0}$, we see that $T x_{0} \neq 0$. Consider $n_{T}, M_{T}, n_{\varphi(T)}$ and $M_{\varphi(T)}$ given respectively by (3.7) and (3.8), and note that $n_{T}=2$ and $M_{T}$ is spanned by $x_{0}$ and $T x_{0}$. Since $\mathrm{r}_{T}\left(x_{0}\right)=0, \mathrm{r}_{\varphi(T)}\left(x_{0}\right)=0$, and therefore (3.5) tells us that $\varphi(T)^{n} x_{0}=0$. Then let $m \geq 3$ be the smallest natural number such that $\varphi(T)^{m}\left(x_{0}\right)=0$. By Lemma 3.5, we have $n_{\varphi(T)}=m$ and

$$
\left\{x_{0}, \varphi(T) x_{0}, \ldots, \varphi(T)^{m-1} x_{0}\right\}
$$

is linearly independent and spanning $M_{\varphi(T)}$. Pick then a linear functional $f$ on $\mathbb{C}^{n}$ such that

$$
f\left(x_{0}\right)=f\left(\varphi(T) x_{0}\right)=\cdots=f\left(\varphi(T)^{m-2} x_{0}\right)=0
$$

and $f\left(\varphi(T)^{m-1} x_{0}\right)=1$, and denote $R_{0}:=x_{0} \otimes f$. Since $f\left(x_{0}\right)=0$, Step 3 tells us that there is a nonzero scalar $\alpha \in \mathbb{C}$ such that $\varphi\left(R_{0}\right)=\alpha R_{0}$. That $R_{0} x_{0}=$ $R_{0}\left(\varphi(T) x_{0}\right)=0$ and $\varphi(T) x_{0}=T x_{0}$ imply that $R_{0}$ is identically zero on $M_{T}$, and therefore by Lemma 3.6, we obtain $r_{T+R_{0}}\left(x_{0}\right)=0$. Then $r_{\varphi(T)+\varphi\left(R_{0}\right)}\left(x_{0}\right)=0$; that is, $\mathrm{r}_{\varphi(T)+\alpha R_{0}}\left(x_{0}\right)=0$. By our construction, we have $\left(\varphi(T)+\alpha R_{0}\right)^{m} x_{0}=\alpha x_{0}$, and thus $0=\mathrm{r}_{\varphi(T)+\alpha R_{0}}\left(x_{0}\right)=|\alpha|$. This contradicts the fact that $\alpha \neq 0$, and shows that $\varphi(T)^{2} x_{0}=0$, as desired.

Step 5 There are two linear maps $v: \mathcal{M}_{n-1,1} \rightarrow \mathcal{M}_{1, n-1}$ and $w: \mathcal{M}_{n-1,1} \rightarrow \mathcal{M}_{n-1}$ such that

$$
v(c) c=0, w(c) c=0 \quad\left(c \in \mathcal{M}_{n-1,1}\right)
$$

and

$$
\varphi\left(\begin{array}{ll}
a & b \\
c & d
\end{array}\right)=\left(\begin{array}{cc}
a & a b_{0}+b+v(c)+\sum \beta_{j} d_{j} \\
c & a(\beta-1) I_{n-1}+w(c)+\beta d
\end{array}\right) \quad\left(\left(\begin{array}{ll}
a & b \\
c & d
\end{array}\right) \in \mathcal{M}_{n}\right)
$$

where $\beta$ is a nonzero complex number and $b_{0}=\left(\beta_{1}, \ldots, \beta_{n-1}\right)$.

There are two linear maps $v: \mathcal{M}_{n-1,1} \rightarrow \mathcal{M}_{1, n-1}$ and $w: \mathcal{M}_{n-1,1} \rightarrow \mathcal{M}_{n-1}$ such that

$$
\varphi\left(\begin{array}{ll}
0 & 0 \\
c & 0
\end{array}\right)=\left(\begin{array}{ll}
0 & v(c) \\
c & w(c)
\end{array}\right) \quad\left(c \in \mathcal{M}_{n-1,1}\right)
$$

Since $\left(\begin{array}{ll}0 & 0 \\ c & 0\end{array}\right)^{2} x_{0}=0$, the previous step tells us that $\left(\begin{array}{ll}0 & v(c) \\ c & w(c)\end{array}\right)^{2} x_{0}=0$, and therefore

$$
v(c) c=0, w(c) c=0 \quad\left(c \in \mathcal{M}_{n-1,1}\right)
$$


For $T=\left(\begin{array}{ll}a & b \\ c & d\end{array}\right) \in \mathcal{M}_{n}$, from (4.2) and (6.8), we infer that

$$
\left\{\begin{array} { l } 
{ a ^ { 2 } + b c = 0 } \\
{ a c + d c = 0 \in \mathcal { M } _ { n - 1 , 1 } }
\end{array} \Longleftrightarrow \left\{\begin{array}{l}
a^{2}+B(T) c=0 \\
a c+D(T) c=0 \in \mathcal{M}_{n-1,1} .
\end{array}\right.\right.
$$

Writing

$$
\varphi\left(\begin{array}{ll}
0 & 0 \\
0 & d
\end{array}\right)=\left(\begin{array}{ll}
0 & u(d) \\
0 & z(d)
\end{array}\right)
$$

with $u: \mathcal{M}_{n-1} \rightarrow \mathcal{M}_{1, n-1}$ and $z: \mathcal{M}_{n-1} \rightarrow \mathcal{M}_{n-1}$ linear maps, (6.4) then gives

$$
\varphi\left(\begin{array}{ll}
a & b \\
c & d
\end{array}\right)=\left(\begin{array}{cc}
a & a b_{0}+\alpha b+v(c)+u(d) \\
c & a d_{0}+w(c)+z(d)
\end{array}\right)
$$

for some $b_{0} \in \mathcal{M}_{1, n-1}$ and $d_{0} \in \mathcal{M}_{n-1}$. Using (6.10) in (6.11), we get

$$
\left\{\begin{array} { l } 
{ a ^ { 2 } + b c = 0 } \\
{ a c + d c = 0 \in \mathcal { M } _ { n - 1 , 1 } }
\end{array} \Longleftrightarrow \left\{\begin{array}{l}
a^{2}+\left(a b_{0}+\alpha b+u(d)\right) c=0 \\
a c+\left(a d_{0}+z(d)\right) c=0 \in \mathcal{M}_{n-1,1} .
\end{array}\right.\right.
$$

Taking $a=0$ and $b=0$ in (6.13), we obtain that

$$
d c=0 \in \mathcal{M}_{n-1,1} \Longleftrightarrow u(d) c=0 \text { and } z(d) c=0 \in \mathcal{M}_{n-1,1}
$$

This and Lemma 3.1 imply the existence of $\beta_{1}, \ldots, \beta_{n-1} \in \mathbb{C}$ such that

$$
u(d)=\sum_{j=1}^{n-1} \beta_{j} d_{j}
$$

where $d_{1}, \ldots, d_{n-1} \in \mathcal{M}_{1, n-1}$ are the rows of any matrix $d \in \mathcal{M}_{n-1}$.

Now, let us show that

$$
z(d)=\left(I_{n-1}+d_{0}\right) d
$$

for all $d \in \mathcal{M}_{n-1}$. First, let us show that if $a \in \mathbb{C}$ and $d \in \mathcal{M}_{n-1}$, then

$$
\operatorname{det}\left(a I_{n-1}+d\right)=0 \Longleftrightarrow \operatorname{det}\left(a\left(I_{n-1}+d_{0}\right)+z(d)\right)=0 .
$$

Indeed, if $-a$ is an eigenvalue of a matrix $d \in \mathcal{M}_{n-1}$, there exists a nonzero matrix $c$ in $\mathcal{M}_{n-1,1}$ such that $a c+d c=0$. Let then $b \in \mathcal{M}_{1, n-1}$ such that $a^{2}+b c=0$, and then (6.13) gives $a c+a d_{0} c+z(d) c=0$. Thus, $\operatorname{det}\left(a I_{n-1}+d\right)=0$ implies that $\operatorname{det}\left(a\left(I_{n-1}+d_{0}\right)+z(d)\right)=0$. The converse follows in the same way, and thus (6.17) always holds. Second, we show that $I_{n-1}+d_{0} \in \mathcal{M}_{n-1}$ is invertible. If not, let $c_{0}$ be a nonzero matrix in $\mathcal{M}_{n-1,1}$ such that $\left(I_{n-1}+d_{0}\right) c_{0}=0$. It then follows from (6.14) that $z\left(I_{n-1}+d_{0}\right) c_{0}=0$, and therefore $\left(a\left(I_{n-1}+d_{0}\right)+z\left(I_{n-1}+d_{0}\right)\right) c_{0}=0$ for all $a \in \mathbb{C}$. This and (6.17) imply that $\operatorname{det}\left((a+1) I_{n-1}+d_{0}\right)=0$ for all $a \in \mathbb{C}$, which in its turn arises a contradiction and shows that $I_{n-1}+d_{0} \in \mathcal{M}_{n-1}$ invertible, as claimed. Finally, we show that the linear map $z$ has the desired form. From (6.17), we see that

$$
\operatorname{det}\left(a I_{n-1}+d\right)=0 \Longleftrightarrow \operatorname{det}\left(a I_{n-1}+\left(I_{n-1}+d_{0}\right)^{-1} z(d)\right)=0 \text {. }
$$

This means that $d \mapsto\left(I_{n-1}+d_{0}\right)^{-1} z(d)$ is a linear map on $\mathcal{M}_{n-1}$ that preserves the spectrum. By [35], there exists an invertible $q \in \mathcal{M}_{n-1}$ such that either $z(d)=\left(I_{n-1}+\right.$ $\left.d_{0}\right) q d q^{-1}$ for all $d \in \mathcal{M}_{n-1}$, or $z(d)=\left(I_{n-1}+d_{0}\right) q d^{t} q^{-1}$ for all $d \in \mathcal{M}_{n-1}$. If the first case occurs, then $d c=0$ implies $d q^{-1} c=0$. This gives $q \in \mathbb{C} I_{n-1}$, and therefore $z(d)=\left(I_{n-1}+d_{0}\right) d$ for each $d$. Since $n>2$, the second case cannot occur. Indeed, 
$d c=0$ would then imply $d^{t} q^{-1} c=0$. Let $c \neq 0$, a nonzero linear functional $f$ on $\mathbb{C}^{n-1}$ such that $f(\bar{c})=0$ and put $\bar{d}=\left(q^{-1} c\right) \otimes f$. We have that $d c$ is zero, but $d^{t} q^{-1} c$ is not!

Next, let us show that

$$
\alpha=1 \text { and } b_{0}=\left(\beta_{1}, \ldots, \beta_{n-1}\right),
$$

where $\beta_{1}, \ldots, \beta_{n-1}$ are the scalars involved in (6.15). Write $b_{0}=\left(\delta_{1}, \ldots, \delta_{n-1}\right)$, and let us prove that $\alpha=1$ and $\delta_{j}=\beta_{j}$ for all $j$. Given any $a \in \mathbb{C}$ and nonzero $c \in \mathcal{M}_{n-1,1}$, there exists $d \in \mathcal{M}_{n-1}$ such that $a c+d c=0$. Then let $b \in \mathcal{M}_{1, n-1}$ such that $a^{2}+b c=0$, and note that, since $b c=-a^{2}$ and $d_{j} c=-a c_{j}$ for each $j$, equations (6.13) and (6.15) imply that

$$
\begin{aligned}
0 & =a^{2}+\left(a b_{0}+\alpha b+u(d)\right) c \\
& =a^{2}+\alpha b c+a \sum_{j=1}^{n-1} \delta_{j} c_{j}+\sum_{j=1}^{n-1} \beta_{j} d_{j} c \\
& =a^{2}(1-\alpha)+a \sum_{j=1}^{n-1}\left(\delta_{j}-\beta_{j}\right) c_{j} .
\end{aligned}
$$

Since $a \in \mathbb{C}$ and $c \in \mathcal{M}_{n-1,1} \backslash\{0\}$ are arbitrary, we conclude that $\alpha=1$ and $\delta_{j}=\beta_{j}$ for all $j=1, \ldots, n-1$; as desired.

Denote $\beta:=I_{n-1}+d_{0}$, and let us prove that $\beta$ is a scalar matrix in $\mathcal{M}_{n-1}$. Let $c \in \mathcal{M}_{n-1,1}$ and $d \in \mathcal{M}_{n-1}$ be a nilpotent matrix, and set $T:=\left(\begin{array}{ll}0 & 0 \\ c & d\end{array}\right)$. Note that since $\mathrm{r}_{d}(c)=0$ in $\mathcal{M}_{n-1}$, we have $\mathrm{r}_{T}\left(x_{0}\right)=0$ and thus $(\varphi(T))^{n} x_{0}=0$, by (3.5). In view of (6.12), (6.15), and (6.16), we have $\varphi(T)=\left(\begin{array}{cc}0 & v(c)+\sum \beta_{j} d_{j} \\ c & w(c)+\beta d\end{array}\right)$, and thus after replacing $d$ by $\lambda d$, one has

$$
\left(\left(\begin{array}{ll}
0 & v(c) \\
c & w(c)
\end{array}\right)+\lambda\left(\begin{array}{cc}
0 & \sum \beta_{j} d_{j} \\
0 & \beta d
\end{array}\right)\right)^{n} x_{0}=0 \quad(\lambda \in \mathbb{C})
$$

Then Lemma 3.8 gives

$$
\left(\begin{array}{cc}
0 & \sum \beta_{j} d_{j} \\
0 & \beta d
\end{array}\right)^{n-1}\left(\begin{array}{cc}
0 & v(c) \\
c & w(c)
\end{array}\right) x_{0}=0
$$

In particular, $(\beta d)^{n-1} c=0$. Since $c \in \mathcal{M}_{n-1,1}$ is an arbitrary matrix, we conclude that $(\beta d)^{n-1}=0$. Therefore, $d \in \mathcal{M}_{n-1}$ nilpotent implies $\beta d \in \mathcal{M}_{n-1}$ nilpotent. The main result of [10] implies the existence of a complex scalar $\delta$ and an invertible $q \in \mathcal{M}_{n-1}$ such that either $\beta d=\delta q d q^{-1}$ for every $d$ having its trace equal to zero, or $\beta d=$ $\delta q d^{t} q^{-1}$ for every $d$ with zero trace. In both cases we have that $\operatorname{tr}(d)=0$ implies $\operatorname{tr}(\beta d)=0$. This implies that $\beta$ is a scalar matrix, as claimed. (Since $\beta$ is scalar, in fact, the second case cannot occur).

Finally, we are in position to conclude and finish the proof of this step. By what has been shown previously, there is a, nonzero scalar $\beta \in \mathbb{C}$ such that $d_{0}=(\beta-1) I_{n-1}$ and thus (6.16) becomes $z(d)=\beta d$ for all $d \in \mathcal{M}_{n-1}$. This together with (6.12), (6.15), and (6.18) show that $\varphi$ has the form (6.9), and thus the proof of this step is complete.

Step 6 For every $T \in \mathcal{M}_{n}$, we have

$$
T^{3}\left(x_{0}\right)=0 \Longleftrightarrow \varphi(T)^{3}\left(x_{0}\right)=0 .
$$


Let us observe that for $T=\left(\begin{array}{ll}a & b \\ c & d\end{array}\right) \in \mathcal{M}_{n}$, we have

$$
T^{2}\left(x_{0}\right)=\left(\begin{array}{l}
a^{2}+b c \\
a c+d c
\end{array}\right)
$$

and

$$
\varphi(T)^{2}\left(x_{0}\right)=\left(\begin{array}{c}
a^{2}+b c+a \sum \beta_{j} c_{j}+\sum \beta_{j} d_{j} c \\
\beta(a c+d c)
\end{array}\right),
$$

by (6.9). This shows, in particular, that $\varphi(T)^{2}\left(x_{0}\right)-\beta T^{2}\left(x_{0}\right) \in \mathbb{C} x_{0}$. Now, suppose for the sake of contradiction that $T^{3} x_{0}$ is 0 , but $\varphi(T)^{3}\left(x_{0}\right)$ is not. We proceed as in the proof of Step 4, and let $m \geq 4$ be the smallest natural number such that $\varphi(T)^{m}\left(x_{0}\right)=0$ and $f$ a linear functional $f$ on $\mathbb{C}^{n}$ such that

$$
f\left(x_{0}\right)=f\left(\varphi(T) x_{0}\right)=\cdots=f\left(\varphi(T)^{m-2} x_{0}\right)=0
$$

and $f\left(\varphi(T)^{m-1} x_{0}\right)=1$. Since $\varphi(T) x_{0}=T x_{0}$ and $\varphi(T)^{2}\left(x_{0}\right)-\beta T^{2}\left(x_{0}\right) \in \mathbb{C} x_{0}$ with $\beta \neq 0$, we have $f\left(x_{0}\right)=f\left(T x_{0}\right)=f\left(T^{2} x_{0}\right)=0$. Denoting $R_{0}=x_{0} \otimes f, R_{0}$ is then identically zero on $M_{T}$, and now, as in the proof of Step 4, we arrive at a contradiction. This contradiction shows that $T^{3} x_{0}=0$ implies that $\varphi(T)^{3}\left(x_{0}\right)=0$, and then, working with $\varphi^{-1}$ instead of $\varphi$, the reverse implication holds as well.

Step 7 The map $\varphi$ given by (6.9) is the identity; i.e., $v=w \equiv 0, \beta=1$, and $\beta_{j}=0$ for all $j=1, \ldots, n-1$.

Let $c \in \mathcal{M}_{n-1,1}$ be a nonzero matrix and pick up $x \in \mathcal{M}_{n-1,1}$ such that $x$ and $c$ are linearly independent. Let $d \in \mathcal{M}_{n-1}$ such that $d c=x$ and $d x=0$, and then $d^{2} c=0$. For $T=\left(\begin{array}{ll}0 & 0 \\ c & d\end{array}\right) \in \mathcal{M}_{n}$, we have

$$
\varphi(T)=\left(\begin{array}{cc}
0 & v(c)+\sum \beta_{j} d_{j} \\
c & w(c)+\beta d
\end{array}\right) \quad \text { and } \quad \varphi(T)^{2} x_{0}=\left(\begin{array}{c}
\sum \beta_{j} d_{j} c \\
\beta d c
\end{array}\right)
$$

see (6.9) and (6.20). Note that since $d^{2} c=0$, we have $T^{3} x_{0}=0$ and (6.19) implies that

$$
0=\varphi(T)^{3}\left(x_{0}\right)=\left(\begin{array}{cc}
0 & v(c)+\sum \beta_{j} d_{j} \\
c & w(c)+\beta d
\end{array}\right)\left(\begin{array}{c}
\sum \beta_{j} d_{j} c \\
\beta d c
\end{array}\right)=\left(\begin{array}{c}
\beta\left(v(c)+\sum \beta_{j} d_{j}\right) d c \\
c\left(\sum \beta_{j} d_{j}\right) c+\beta w(c) d c
\end{array}\right) .
$$

Since $d^{2} c=0$, we have $d_{j} d c=0$ for all $j$, and thus

$$
0=\left(v(c)+\sum \beta_{j} d_{j}\right) d c=v(c) d c=v(c) x
$$

and

$$
0=c\left(\sum \beta_{j} d_{j}\right) c+\beta w(c) d c=c\left(\sum \beta_{j} d_{j}\right) c+\beta w(c) x .
$$

From (6.21), we conclude that $v \equiv 0$.

Now, note that, since $d_{j} c=x_{j}$ for all $j=1, \ldots, n-1$, equation (6.22) becomes

$$
\left(\sum \beta_{j} x_{j}\right) c+\beta w(c) x=0
$$

By continuity, this holds for $x=c$ too, and since $w(c) c=0$, we get $\sum \beta_{j} c_{j}=0$ for every nonzero $c \in \mathcal{M}_{n-1,1}$. Thus, $\beta_{j}=0$ for all $j$. Then (6.23) gives $w(c) x=0$ for each $c$ and $x$ that are linearly independent, and this implies $w \equiv 0$. 
Thus, (6.9) becomes

$$
\varphi\left(\begin{array}{ll}
a & b \\
c & d
\end{array}\right)=\left(\begin{array}{lc}
a & b \\
c & a(\beta-1) I_{n-1}+\beta d
\end{array}\right) \quad\left(\left(\begin{array}{ll}
a & b \\
c & d
\end{array}\right) \in \mathcal{M}_{n}\right)
$$

For

$$
T=\left(\begin{array}{ccccc}
-1 & 0 & 1 & 0 & \ldots \\
1 & 0 & -1 & 0 & \ldots \\
0 & 1 & 1 & 0 & \ldots \\
0 & 0 & 0 & 0 & \ldots \\
\vdots & \vdots & \vdots & \vdots & \ddots
\end{array}\right)
$$

we have $T^{3}=0$ and

$$
\varphi(T)=\left(\begin{array}{ccccc}
-1 & 0 & 1 & 0 & \ldots \\
1 & 1-\beta & -\beta & 0 & \ldots \\
0 & \beta & 1 & 0 & \ldots \\
0 & 0 & 0 & 1-\beta & \ldots \\
\vdots & \vdots & \vdots & \vdots & \ddots
\end{array}\right) .
$$

By (6.19), we have

$$
0=\varphi(T)^{3} x_{0}=(\beta-1)\left(\begin{array}{c}
1 \\
-1 \\
-\beta \\
0 \\
\vdots
\end{array}\right) .
$$

Clearly, $\beta=1$, and the proof is therefore complete.

\section{Concluding Remarks and Open Problems}

In the sequel, let $X$ and $Y$ be two complex Banach spaces, and let $x_{0} \in X$ and $y_{0} \in Y$ be two nonzero vectors. In this section, we make some remarks and comments on linear and nonlinear preservers of local spectral radius and discuss some further challenging problems, which are suggested by the main results of this paper. First, we wonder if our main results remain valid for the infinite-dimensional case.

Problem 1 Which linear maps $\varphi$ from $\mathcal{L}(X)$ onto $\mathcal{L}(Y)$ satisfy

$$
\mathrm{r}_{T}\left(x_{0}\right)=0 \Longleftrightarrow \mathrm{r}_{\varphi(T)}\left(y_{0}\right)=0 \quad(T \in \mathcal{L}(X)) ?
$$

When $X$ and $Y$ are infinite-dimensional Banach spaces, we conjecture that a linear map $\varphi$ from $\mathcal{L}(X)$ onto $\mathcal{L}(Y)$ satisfies (7.1) if and only if there is a nonzero scalar $\alpha \in \mathbb{C}$ and a bijective bounded linear mapping $A$ from $X$ into $Y$ such that $A x_{0}=y_{0}$ and $\varphi(T)=\alpha A T A^{-1}$ for all $T \in \mathcal{L}(X)$. Note that the injectivity of any linear map $\varphi$ satisfying (7.1) follows from [16, Theorem 3.1]. But, unlike for the finite-dimensional case, the surjectivity assumption of such a map $\varphi$ is necessary. Otherwise, the description of maps $\varphi$ from $\mathcal{L}(X)$ into $\mathcal{L}(Y)$ satisfying (7.1) would be vague, since the mapping $\varphi$ from $\mathcal{L}(X)$ into $\mathcal{L}(X \oplus X)$, defined by $\varphi(T)=T \oplus T,(T \in \mathcal{L}(X))$, satisfies (7.1) when $y_{0}=x_{0} \oplus x_{0}$. 
We also would like to point out that the full description of all linear maps $\varphi$ from $\mathcal{L}(X)$ onto $\mathcal{L}(Y)$ satisfying

$$
\mathrm{r}_{\varphi(T)}\left(y_{0}\right)=\mathrm{r}_{T}\left(x_{0}\right) \quad(T \in \mathcal{L}(X)) .
$$

is well known and can be found in [13,18,20,26]. In [20], Bračič and Müller extended the main result of [18] to infinite-dimensional Banach spaces by characterizing surjective linear and continuous maps on $\mathcal{L}(X)$ that preserve the local spectral radius at a fixed nonzero vector of $X$. In [26, Theorem 1.2], Costara showed that surjective linear local spectral radius preservers on $\mathcal{L}(X)$ are automatically continuous.

A number of authors studied maps preserving the spectra and local spectra of different products of matrices or operators that includes the usual product, the triple product and the Jordan product; see, for instance, [11-16, 23, 25, 27,31,32]. It is also worth mentioning that the full description of surjective maps $\varphi$ from $\mathcal{L}(X)$ into $\mathcal{L}(Y)$ preserving the spectral radius of different products of operators is unknown; see [13]. As far as for the nonlinear local spectral radius preservers, we first state the following problem.

Problem 2 Which maps $\varphi$ from $\mathcal{L}(X)$ onto $\mathcal{L}(Y)$ satisfy

$$
\mathrm{r}_{S \pm T}\left(x_{0}\right)=0 \Longleftrightarrow \mathrm{r}_{\varphi(S) \pm \varphi(T)}\left(y_{0}\right)=0 \quad(S, T \in \mathcal{L}(X)) ?
$$

Obviously, (7.2) holds for any map $\varphi$ from $\mathcal{L}(X)$ onto $\mathcal{L}(Y)$ satisfying

$$
\mathrm{r}_{\varphi(S) \pm \varphi(T)}\left(y_{0}\right)=\mathrm{r}_{S_{ \pm} T}\left(x_{0}\right) \quad(S, T \in \mathcal{L}(X)) .
$$

In the finite-dimensional case, the description of such maps is known as shown by Costara in [23]. He proved that a surjective map $\varphi$ on $\mathcal{M}_{n}$ satisfies (7.3) with $\varphi(0)=0$ if and only if $\varphi$ is an automorphism multiplied by a scalar of modulus one and the intertwining matrix sends $x_{0}$ to $y_{0}$. However, when $X$ and $Y$ are infinite-dimensional spaces, the characterization of maps satisfying (7.3) is unknown and remains an open problem as well.

In [14], Bourhim and Mashreghi showed that a map $\varphi$ from $\mathcal{L}(X)$ onto $\mathcal{L}(Y)$ satisfies

$$
\sigma_{\varphi(T) \varphi(S)}\left(y_{0}\right)=\sigma_{T S}\left(x_{0}\right) \quad(T, S \in \mathcal{L}(X))
$$

if and only if there exists a bijective bounded linear mapping $A$ from $X$ into $Y$ such that $A x_{0}=y_{0}$ and either $\varphi(T)=A T A^{-1}$ for all $T \in \mathcal{L}(X)$ or $\varphi(T)=-A T A^{-1}$ for all $T \in \mathcal{L}(X)$. Naturally, this result suggests the problem of describing all maps $\varphi$ from $\mathcal{L}(X)$ onto $\mathcal{L}(Y)$ for which

$$
\mathrm{r}_{\varphi(T) \varphi(S)}\left(y_{0}\right)=\mathrm{r}_{T S}\left(x_{0}\right) \quad(T, S \in \mathcal{L}(X)) .
$$

Even more, one may ask the following more general question of describing all maps on $\mathcal{L}(X)$ preserving the product of operators of local spectral radius zero at some fixed nonzero vector of $X$.

Problem 3 Which maps $\varphi$ from $\mathcal{L}(X)$ onto $\mathcal{L}(Y)$ satisfy

$$
\mathrm{r}_{S T}\left(x_{0}\right)=0 \Longleftrightarrow \mathrm{r}_{\varphi(S) \varphi(T)}\left(y_{0}\right)=0 \quad(S, T \in \mathcal{L}(X)) \text { ? }
$$


Similar questions can be asked when replacing the usual product by triple or Jordan product.

Problem 4 Describe all maps $\varphi$ from $\mathcal{L}(X)$ onto $\mathcal{L}(Y)$ satisfying either

$$
\mathrm{r}_{S T S}\left(x_{0}\right)=0 \Longleftrightarrow \mathrm{r}_{\varphi(S) \varphi(T) \varphi(S)}\left(y_{0}\right)=0 \quad(S, T \in \mathcal{L}(X))
$$

or

$$
\mathrm{r}_{S T+T S}\left(x_{0}\right)=0 \Longleftrightarrow \mathrm{r}_{\varphi(S) \varphi(T)+\varphi(T) \varphi(S)}\left(y_{0}\right)=0 \quad(S, T \in \mathcal{L}(X)) .
$$

One may ask similar questions for different local spectra, in particular, when the local spectral radius is replaced by the inner local spectral radius. For a positive scalar $r$, let $\overline{\mathrm{D}}(0, r)$ (resp. $\mathrm{D}(0, r)$ ) denote the closed (resp. the open) disc centered at the origin with radius $r$, and for a closed subset $F$ of $\mathbb{C}$ and an operator $T \in \mathcal{L}(X)$, the subspace

$$
X_{T}(F):=\{x \in X:(T-\lambda) f(\lambda)=x \text { has an analytic solution } f \text { on } \mathbb{C} \backslash F\}
$$

is the so-called glocal spectral subspace of $T$. Recall that the local spectral radius of $T$ at any vector $x \in X$ coincides with

$$
\mathrm{r}_{T}(x)=\inf \left\{r \geq 0: x \in X_{T}(\overline{\mathrm{D}}(0, r))\right\}
$$

see [34, Proposition 3.3.13]. Analogously, the inner local spectral radius of $T$ at $x$ is defined by

$$
\iota_{T}(x):=\sup \left\{r \geq 0: x \in X_{T}(\mathbb{C} \backslash \mathrm{D}(0, r))\right\},
$$

and note that $\iota_{T}(x)=0$ if and only if $0 \in \sigma_{T}(x)$; see [36]. We also note that if $T \in \mathcal{M}_{n}$ is a matrix and $x \in \mathbb{C}^{n}$, then $\iota_{T}(x)$ is nothing but the minimum modulus of $\sigma_{T}(x)$.

Problem 5 Which linear maps $\varphi$ from $\mathcal{L}(X)$ onto $\mathcal{L}(Y)$ satisfy

$$
\iota_{T}\left(x_{0}\right)=0 \Longleftrightarrow \iota_{\varphi(T)}\left(y_{0}\right)=0 \quad(T \in \mathcal{L}(X)) ?
$$

Consider a unital map $\varphi$ from $\mathcal{L}(X)$ onto $\mathcal{L}(Y)$ satisfying (7.4). Then for every $T \in \mathcal{L}(X)$, we have

$$
\begin{aligned}
\lambda \in \sigma_{T}\left(x_{0}\right) & \Longleftrightarrow \iota_{T-\lambda}\left(x_{0}\right)=0 \\
& \Longleftrightarrow \iota_{\varphi(T)-\lambda}\left(y_{0}\right)=0 \\
& \Longleftrightarrow \lambda \in \sigma_{\varphi(T)}\left(y_{0}\right) .
\end{aligned}
$$

This implies that

$$
\sigma_{\varphi(T)}\left(y_{0}\right)=\sigma_{T}\left(x_{0}\right)
$$

for all $T \in \mathcal{L}(X)$. Therefore, by [20,22], there is a bijective bounded linear mapping $A$ from $X$ into $Y$ such that $A x_{0}=y_{0}$ and $\varphi(T)=A T A^{-1}$ for all $T \in \mathcal{L}(X)$. So, to answer the last question, one only needs to determine $\varphi(\mathbf{1})$ when $\varphi$ satisfies (7.4). 


\section{References}

[1] P. Aiena, Fredholm and local spectral theory, with applications to multipliers. Kluwer Academic Publishers, Dordrecht, 2004.

[2] J. Alaminos, M. Brešar, P. Šemrl, and A. R. Villena, A note on spectrum-preserving maps. J. Math. Anal. Appl. 387(2012), 595-603. http://dx.doi.org/10.1016/j.jmaa.2011.09.024

[3] J. Alaminos, J. Extremera, and A. R. Villena, Approximately spectrum-preserving maps. J. Funct. Anal. 261(2011), 233-266. http://dx.doi.org/10.1016/j.jfa.2011.02.020

[4] J. Alaminos, M. Brešar, J. Extremera, and A. R. Villena, Maps preserving zero products. Studia Math. 193(2009), 131-159. http://dx.doi.org/10.4064/sm193-2-3

[5] B. Aupetit, Spectrum-preserving linear mappings between Banach algebras or Jordan-Banach algebras. J. London Math. Soc. 62(2000), 917-924. http://dx.doi.org/10.1112/S0024610700001514

[6] Sur les transformations qui conservent le spectre. In: Banach algebras 97 (Blaubeuren), de Gruyter, Berlin, 1998, 55-78.

[7] B. Aupetit and H. T. Mouton, Spectrum preserving linear mappings in Banach algebras. Studia Math. 109(1994), 91-100. http://dx.doi.org/10.4064/sm-109-1-91-100

[8] L. Baribeau and T. Ransford, Non-linear spectrum-preserving maps. Bull. London Math. Soc. 32(2000), 8-14. http://dx.doi.org/10.1112/S0024609399006426

[9] R. Bhatia, P. Šemrl, and A. Sourour, Maps on matrices that preserve the spectral radius distance. Studia Math. 134(1999), 99-110.

[10] P. Botta, S. Pierce, and W. Watkins, Linear transformations that preserve the nilpotent matrices. Pacific J. Math. 104(1983), 39-46. http://dx.doi.org/10.2140/pjm.1983.104.39

[11] A. Bourhim and M. Mabrouk, Jordan product and local spectrum preservers. Studia Math. 234(2016), 97-120.

[12] Maps preserving the local spectrum of Jordan product of matrices. Linear Algebra Appl. 484(2015), 379-395. http://dx.doi.org/10.1016/j.laa.2015.06.034

[13] A. Bourhim and J. Mashreghi, A survey on preservers of spectra and local spectra. In: Invariant subspaces of the shift operator, Contemp. Math., 638, American Mathematical Society, Providence, RI, 2015, pp. 45-98. http://dx.doi.org/10.1090/conm/638/12810

[14] Maps preserving the local spectrum of product of operators. Glasgow Math. J. 57(2015), 709-718. http://dx.doi.org/10.1017/S0017089514000585

[15] _ Maps preserving the local spectrum of triple product of operators. Linear Multilinear Algebra 63(2015), 765-773. http://dx.doi.org/10.1080/03081087.2014.898299

[16] Local spectral radius preservers. Integral Equations Operator Theory 76(2013), 95-104 http://dx.doi.org/10.1007/s00020-013-2041-9

[17] A. Bourhim, M. Burgos, and V. S. Shulman, Linear maps preserving the minimum and reduced minimum moduli. J. Funct. Anal. 258(2010), 50-66. http://dx.doi.org/10.1016/j.jfa.2009.10.003

[18] A. Bourhim and V. Miller, Linear maps on $\mathcal{M}_{n}(\mathbb{C})$ preserving the local spectral radius. Studia Math. 188(2008), 67-75. http://dx.doi.org/10.4064/sm188-1-4

[19] A. Bourhim and T. Ransford, Additive maps preserving local spectrum. Integral Equations Operator Theory 55(2006), 377-385. http://dx.doi.org/10.1007/s00020-005-1392-2

[20] J. Bračič and V. Müller, Local spectrum and local spectral radius of an operator at a fixed vector. Studia Math. 194(2009), 155-162. http://dx.doi.org/10.4064/sm194-2-3

[21] M. Brešar and P. Šemrl, Linear maps preserving the spectral radius. J. Funct. Anal. 142(1996), 360-368. http://dx.doi.org/10.1006/jfan.1996.0153

[22] C. Costara, Automatic continuity for linear surjective maps compressing the local spectrum at fixed vectors, Proc. Amer. Math. Soc., 145, No. 5, (2017) 2081-2087. http://dx.doi.org/10.1090/proc/13364

[23] _ Surjective maps on matrices preserving the local spectral radius distance. Linear Multilinear Algebra 62(2014), 988-994. http://dx.doi.org/10.1080/03081087.2013.801967

[24] L Linear maps preserving operators of local spectral radius zero. Integral Equations Operator Theory 73(2012), 7-16. http://dx.doi.org/10.1007/s00020-012-1953-0

[25] $\longrightarrow$ Maps on matrices that preserve the spectrum. Linear Algebra Appl. 435(2011), 2674-2680. http://dx.doi.org/10.1016/j.laa.2011.04.026

[26] _ Automatic continuity for linear surjective mappings decreasing the local spectral radius at some fixed vector. Arch. Math. 95(2010), 567-573. http://dx.doi.org/10.1007/s00013-010-0191-4

[27] C. Costara and D. Repovš, Nonlinear mappings preserving at least one eigenvalue. Studia Math. 200(2010), 79-89. http://dx.doi.org/10.4064/sm200-1-5

[28] J. Dieudonné, Sur une généralisation du groupe orthogonal a quatre variables. Arch. Math. 1(1949), 282-287. http://dx.doi.org/10.1007/BF02038756 
[29] H. Flanders, On spaces of linear transformations with bounded rank. J. London Math. Soc. 37(1962), 10-16. http://dx.doi.org/10.1112/jlms/s1-37.1.10

[30] G. Frobenius, Ueber die Darstellung der endlichen Gruppen durch lineare Substitutionen. Berl. Ber. (1897), 994-1015.

[31] J. C. Hou, C. K. Li, N. C. Wong, Maps preserving the spectrum of generalized Jordan product of operators. Linear Algebra Appl. 432(2010), 1049-1069. http://dx.doi.org/10.1016/j.laa.2009.10.018

[32] - Jordan isomorphisms and maps preserving spectra of certain operator products. Studia Math. 184(2008), 31-47. http://dx.doi.org/10.4064/sm184-1-2

[33] A. A. Jafarian and A. R. Sourour, Spectrum-preserving linear maps. J. Funct. Anal. 66(1986), 255-261. http://dx.doi.org/10.1016/0022-1236(86)90073-X

[34] K. B. Laursen and M. M. Neumann, An introduction to local spectral theory. London Mathematical Society Monographs, New Series, 20, The Clarendon Press, Oxford University Press, New York, 2000.

[35] M. Marcus and B. N. Moyls, Linear transformations on algebras of matrices. Canad. J. Math. 11(1959), 61-66. http://dx.doi.org/10.4153/CJM-1959-008-0

[36] T. L. Miller, V. G. Miller, and M. M. Neumann, Local spectral properties of weighted shifts. J. Operator Theory 51(2004), 71-88.

[37] L. Molnár and M. Barczy, Linear maps on the space of all bounded observables preserving maximal deviation. J. Funct. Anal. 205(2003), 380-400. http://dx.doi.org/10.1016/S0022-1236(03)00213-1

[38] L. Molnár, Orthogonality preserving transformations on indefinite inner product spaces: generalization of Uhlhorn's version of Wigner's theorem. J. Funct. Anal. 194(2002), 248-262. http://dx.doi.org/10.1006/jfan.2002.3970

[39] P. Šemrl, Linear maps that preserve the nilpotent operators. Acta Sci. Math. 61(1995), 523-534.

[40] A. R. Sourour, Invertibility preserving linear maps on $\mathcal{L}(X)$. Trans. Amer. Math. Soc. 348(1996), 13-30. http://dx.doi.org/10.1090/S0002-9947-96-01428-6

[41] A. Torgašev, On operators with the same local spectra. Czechoslovak Math. J. 48(1998), 77-83. http://dx.doi.org/10.1023/A:1022467611697

Department of Mathematics, Syracuse University, Syracuse, NY 13244, USA

e-mail: abourhim@syr.edu

Faculty of Mathematics and Informatics, Ovidius University of Constanţa, Mamaia Boul. 124, 900527, Constanţa, Romania

e-mail: cdcostara@univ-ovidius.ro 